\title{
TOLLMIEN-SCHLICHTING WAVE AMPLITUDES ON A SEMI-INFINITE FLAT PLATE AND A PARABOLIC BODY: COMPARISON OF A PARABOLIZED STABILITY EQUATION METHOD AND DIRECT NUMERICAL SIMULATIONS
}

\author{
by M. R. Turner $\ddagger \S$ \\ (Sir Harry Ricardo Laboratories, School of Computing, Engineering and Mathematics, \\ University of Brighton, Lewes Road, Brighton BN2 4 GJ, U.K.)
}

[Received 20 April 2011. Revise 1 November 2011]

\begin{abstract}
Summary
In this paper the interaction of free-stream acoustic waves with the leading edge of an aerodynamic body is investigated and we compare two different methods for analysing this interaction. Results are compared for a method which incorporates Orr-Sommerfeld calculations using the parabolized stability equation (PSE) to those of direct numerical simulations (DNS). By comparing the streamwise amplitude of the Tollmien-Schlichting (T-S) wave it is found that non-modal components of the boundary-layer response to an acoustic wave can persist some distance downstream of the lower branch. The effect of nose curvature on the persisting non-modal eigenmodes is also considered, with a larger nose radius allowing the non-modal eigenmodes to persist farther downstream.
\end{abstract}

\section{Introduction}

For an aerodynamic body placed in a mean flow with a small amplitude unsteady perturbation (either acoustic or vortical), the position of boundary-layer transition depends on both the stability characteristics of the body and how the unsteady disturbance interacts with the boundary layer itself. This interaction process is known as receptivity (1) and is the first stage of the transition process. For two-dimensional, large Reynolds number flows, the transfer of energy from the free-stream disturbance to the instability wave occurs due to non-parallel mean flow effects. These receptivity processes occur at various positions on the body such as at the leading edge $(\mathbf{2}, \mathbf{3})$, or further downstream where the mean flow varies rapidly in the streamwise direction, such as at surface roughness elements $(\mathbf{4}, \mathbf{5}, \mathbf{6}, \mathbf{7}, \mathbf{8})$, regions of marginal stability (9) or changes in surface roughness (10). For receptivity regions upstream of the lower branch point (such as at the leading edge or at a vibrating ribbon), the modal component of the instability wave decays exponentially in amplitude downstream until the lower branch neutral stability point is reached. Beyond the lower branch the disturbance grows exponentially until either nonlinear effects become important and transition occurs or the disturbance decays after reaching the upper branch neutral

$\ddagger$ Current address: Department of Mathematics, University of Surrey, Guildford, Surrey GU2 7XH, U.K.

§ e-mail: m.turner@surrey.ac.uk, tel:+44 1483686183 ,fax:+44 1483686071

Q. Jl Mech. Appl. Math. (2011) xx (y), 1-29

(c) Oxford University Press 2011 
stability point. The work of Saric et al. (11) reviews the asymptotic, numerical and experimental approaches to receptivity and transition.

For instability waves generated at the leading edge of a semi-infinite flat plate via acoustic free-stream disturbances, Goldstein (2) showed that the asymptotic structure of the flow is split into two regions. In the first region, close to the nose of the body, the flow is governed by the unsteady boundary layer equation. The far downstream asymptotic form of the solution in this region consists of a Stokes layer plus a sum of asymptotic eigenmodes $(\mathbf{1 2}, \mathbf{1 3}, \mathbf{1 4})$. The Lam-Rott eigenmodes are important in receptivity analysis because they exhibit wavelength shortening as they move downstream and hence link the longwavelength free-stream disturbances to the much shorter scale instability waves in the boundary layer via a multiplicative receptivity coefficient. This receptivity coefficient has been calculated for a flat plate (15), a parabolic body (16) and a Rankine body (17) via a combination of numerical and asymptotic approaches. The solution in this leading edge region can be asymptotically matched to the large-Reynolds-number, small wavenumber form of the classical Orr-Sommerfeld equation. It has also be shown that the first of the Lam-Rott eigenmodes matches to the unstable Tollmien-Schlichting (T-S) mode of the Orr-Sommerfeld equation, which exhibits exponential streamwise growth downstream (2). A similar asymptotic structure in the leading edge region has been shown to exist on both a parabolic body (16) and more general bodies where the slip velocity tends to a constant downstream (17). An equivalent asymptotic analysis in the Orr-Sommerfeld region for bodies other than the flat plate does not yet exist due to the complex structure in this region. Therefore, the evolution of the $\mathrm{T}-\mathrm{S}$ wave is usually determined via numerical methods.

This paper makes direct comparisons between two different receptivity/stability methods by investigating the spatial evolution of $\mathrm{T}-\mathrm{S}$ waves in the boundary layer generated via acoustic disturbances. One method is conducting direct numerical simulations (DNS) of the T-S wave evolution using the approach examined in Haddad \& Corke (18) and the other method is the parabolized stability equation (PSE) scheme laid out in Turner \& Hammerton (19). The purpose of this comparison is to examine to what extent the PSE scheme describes the streamwise evolution of disturbances in the boundary layer excited by acoustic waves. This is of interest because the receptivity process also excites non-modal eigenmodes from the continuous spectrum of eigenmodes in the boundary layer. these eigenmodes grow or decay algebraically in the streamwise direction $(\mathbf{2 0})$. The PSE method neglects these nonmodal eigenmodes and only follows the evolution of the exponentially growing discrete eigenmodes. One of these discrete eigenmodes is the T-S wave which dominates the boundary-layer solution far downstream. The DNS method on the other hand contains both the modal and non-modal eigenmodes. This paper focuses on determining how far along the body these non-modal eigenmodes persist, and investigates the value of the PSE approach for making comparisons with experiments.

A comparison between the PSE and DNS schemes had previously been considered by Joslin et al. (21) who found excellent agreement between the two methods for the propagation of two-dimensional T-S waves on a semi-infinite flat plate. However, unlike Joslin et al. (21) the PSE method of (19) considered in this paper allows the comparison of T-S wave streamwise velocity amplitudes rather than just growth rates. This is because the PSE method incorporates the asymptotic form of the T-S wave from the leading edge region to give a complete $\mathrm{T}-\mathrm{S}$ wave amplitude. The present study also makes comparisons 
on a parabolic body which will investigate the effect of nose curvature on the boundary layer disturbance evolution. The non-dimensional acoustic frequency $\sigma=\nu \omega^{*} / U_{\infty}^{2}$ used by Joslin et al. (21) was $\sigma=84 \times 10^{-6}$ which is at the lower end of the range used in experiments, therefore here this range is extended to $\sigma \in\left[60 \times 10^{-6}, 230 \times 10^{-6}\right]$ to include a wider range of experimental frequencies.

There have been many numerical studies which have calculated the growth rate of the T-S wave in the Orr-Sommerfeld region for a semi-infinite flat plate. The (parallel flow) Orr-Sommerfeld equation solution has been corrected for non-parallel mean flow effects by expanding the mean flow in powers of $R e^{-1 / 2}$ in the coefficients of the linearized NavierStokes equations $(\mathbf{2 2}, \mathbf{2 3})$. Here $R e$ is the Reynolds number. However, this approach makes the solution more difficult to interpret physically because the $O(1)$ equation of this expansion actually contains terms which are of $O(1), O\left(R e^{-1 / 6}\right), O\left(R e^{-1 / 3}\right)$ and $O\left(R e^{-1 / 2} \ln R e\right)$, as shown by Goldstein (2). Similarly the $O\left(R e^{-1 / 2}\right)$ equation contains separate asymptotic terms too, such as $O\left(R e^{-2 / 3}\right)$ etc, which are combined into one equation. Bertolotti et al. (24) utilized this non-systematic asymptotic approach by combining the non-parallel effects into the single PSE partial differential equation. In the last two decades the PSE has been used within industry, along with other approaches such as the $e^{N}$ method $(\mathbf{7}, \mathbf{2 5})$, and has been extended to take account of hypersonic flows, nonlinearity and chemical reactions within the boundary-layer flow $(\mathbf{2 6}, \mathbf{2 7})$. The main advantage of incorporating the nonparallel effects into one partial differential equation is that the algebra required to eliminate secular terms in numerical computations, such as Saric \& Nayfeh (23), is removed. The PSE has to be integrated downstream from some upstream boundary condition and it is computationally faster than DNS schemes. The PSE had previously been solved using an upstream boundary condition from Orr-Sommerfeld theory or using a local solution to the PSE $(\mathbf{2 4}, \mathbf{2 8})$. However, these approaches did not use the receptivity information at the leading edge to link the free-stream information to the amplitude of the T-S wave. This was overcome by (19) who linked the receptivity information to the T-S wave using a scheme which combined the PSE in the Orr-Sommerfeld region with an upstream boundary condition given by the Lam-Rott eigenmode from the leading edge receptivity analysis. This method allowed the streamwise amplitude of the unstable T-S wave to be calculated. This amplitude was shown to agree with amplitudes calculated via the asymptotic results of $(\mathbf{2})$ in the large Reynolds number limit (29). However, the PSE and asymptotic approaches are not independent, as both methods used the same upstream amplitude condition, and so this comparison only investigated changes in the growth rate between the two methods. Also neither method considered the non-modal eigenmodes within the boundary layer. The present study allows for a comparison of $\mathrm{T}-\mathrm{S}$ wave amplitudes from two independent approaches so we can investigate key differences resulting from the two approaches. The PSE scheme was extended to more general bodies, which have a slip velocity which tends to a constant far from the leading edge, by Turner \& Hammerton (30).

Early DNS studies which investigated the evolution of T-S waves, solved the nonlinear time dependent Navier-Stokes equations, such as in Reed et al. (31). These simulations were conducted to model the experiments of Saric \& Rasmussen (32) and Saric et al. (33) who studied an elliptical leading edge attached to a finite thickness flat plate. The problem with this geometry was that the join between the nose and the plate acted as another region of receptivity due to the discontinuity of curvature at this point. This discontinuity was removed by machining the elliptical nose onto the plate to generate a Modified Super 
Ellipse (MSE). This MSE was investigated numerically by Lin et al. (34). These initial DNS schemes were computationally expensive because they solved the full Navier-Stokes equations. However, experimental evidence suggested that the process of receptivity to the unsteady disturbance was linear. Hence (18) developed a more efficient spatial code which linearised about the base flow and solved for the steady base flow and the unsteady perturbation flow separately. Haddad \& Corke (18) developed their code for a parabolic body so as to eliminate the discontinuous curvature issue of the elliptical body studied by Reed et al. (31), see (10) for a more detailed discussion. This DNS method was extended to parabolic bodies at non-zero angles of attack by Ertuck \& Corke (35) and Haddad et al. (36), and to the MSE by Wanderley \& Corke (37). The results of (37) were compared to the DNS results of Fuciarelli et al. (38) and the experiments of Saric \& White (39). In the present study we use the linearised code approach of (18) as it is computationally faster than the nonlinear Navier-Stokes approach. This method is also preferred because it has been constructed to investigate a parabolic body as well as the flat plate.

The structure of this paper is as follows. In $\S 2.1$ we formulate the PSE scheme including the asymptotic form of the upstream boundary condition, while the DNS method is outlined in $\S 2.2$. Comparative results for a semi-infinite flat plate are given in $\S 3.1$ and for a parabolic body in $\S 3.2$. Conclusions and discussions can be found in $\S 4$.

\section{Formulation}

\subsection{Formulation of the parabolized stability equation scheme}

This section summarizes the derivation of the parabolized stability equation (PSE) scheme which combines PSE calculations in the Orr-Sommerfeld region with an upstream T-S wave amplitude given by the leading edge receptivity analysis. For more information the reader is referred to (19) and (30). The PSE is valid within the boundary layer on a two-dimensional body with a rounded leading edge. In this study we use the coordinate system $\left(x^{*}, y^{*}\right)$ which are dimensional coordinates measured along the body and normal to the body respectively. The dimensionless quantities $\left(x_{P}, y_{P}\right)$ and $t_{P}$ are introduced based on the velocity of the free-stream $U_{\infty}$ and the fixed length scale $\delta_{0}=\left(\nu x_{0}^{*} / U_{\infty}\right)^{1 / 2}$. The vorticity equation written in terms of the stream function $\Psi_{P}$ can then be expressed as

$$
\left(\frac{\partial}{\partial t_{P}}-\frac{1}{R_{0}} \nabla^{2}+\frac{\partial \Psi_{P}}{\partial y_{P}} \frac{\partial}{\partial x_{P}}-\frac{\partial \Psi_{P}}{\partial x_{P}} \frac{\partial}{\partial y_{P}}\right) \nabla^{2} \Psi_{P}=0,
$$

where

$$
R_{0}=\frac{U_{\infty} \delta_{0}}{\nu} .
$$

This equation holds at leading order for general bodies as long as the curvature of the body is assumed to be small away from the vicinity of the leading edge $(\mathbf{4 0}, \mathbf{2 9})$. In (2.1) $\nu$ is the kinematic viscosity, $x_{0}^{*}$ is the dimensional distance along the body at which the PSE analysis is started and $R_{0}$ is the Reynolds number based upon the length scale $\delta_{0}$. The Reynolds number $R_{0}$ is assumed to be large so that the flow field is inviscid and irrotational everywhere, except in the vicinity of the surface of the body. The corresponding non-dimensional position that the numerical analysis is started is $x_{0}=x_{0}^{*} / \delta_{0}=R_{0}$.

The stream function, $\Psi_{P}$, is split into a steady base flow part $\Psi_{B}\left(x_{P}, y_{P}\right)$ and a time dependent disturbance part $\psi_{P}\left(x_{P}, y_{P}, t_{P}\right) \ll \Psi_{B}\left(x_{P}, y_{P}\right)$, and $U_{f}\left(x_{P}\right)$ is defined as the 
mean flow slip velocity at the edge of the boundary layer, parallel to the surface of the body. In order to use the asymptotic leading edge receptivity result formulated in (30) as the upstream boundary condition for the PSE, the coordinate system is changed to

$$
\xi_{P}=\int_{0}^{x_{P}} U_{f}\left(x^{\prime}\right) d x^{\prime}, \quad N_{P}=R_{0}^{1 / 2} U_{f}\left(\xi_{P}\right)\left(2 \xi_{P}\right)^{-1 / 2} y_{P},
$$

which remain in the streamwise and normal directions to the body respectively $(\mathbf{1 7}, \mathbf{2 9})$.

A solution for the disturbance stream function $\psi_{P}\left(\xi_{P}, N_{P}, t_{P}\right)$ is sought in the form of a spatially evolving two-dimensional wave with constant frequency $\omega_{P}$, local streamwise wave number $\alpha\left(\xi_{P}\right)$ and a complex mode shape $\phi_{P}\left(\xi_{P}, N_{P}\right)$ which takes the form

$$
\psi_{P}\left(\xi_{P}, N_{P}, t_{P}\right)=\phi_{P}\left(\xi_{P}, N_{P}\right) \exp \left(i\left(\int_{0}^{\xi_{P}} \alpha\left(\xi_{P}^{\prime}\right) d \xi_{P}^{\prime}-\omega_{P} t_{P}\right)\right)+\text { complex conjugate. }
$$

The amplitude of the disturbance wave is assumed to be sufficiently small so that the non-linear terms can be neglected, $\left|\psi_{P}\right| \ll 1$. This condition is valid up to the lower branch neutral stability point, as the modal disturbance wave generated at the leading edge decays up to this point. This condition is also valid up to the upper branch point as long as the amplification rate of the disturbance is not too large. In this study this is assumed to be the case and PSE calculations are continued to the upper branch. At this point comparisons can be made with the linear DNS where the T-S wave should dominate any non-modal eigenmodes. As direct comparisons with experiments are not made at this stage, this linear approach is an appropriate choice. If $\mathrm{T}-\mathrm{S}$ wave amplitude comparisons with experiments are required at the upper branch, then the nonlinear form of the PSE, which is discussed in Bertolotti et al. (24), should be considered. The main assumption in the formulation of the PSE is that the streamwise variation of both $\alpha$ and $\phi_{P}$ is sufficiently small $(\mathbf{2 4}, \mathbf{1 9})$. This means that $\partial^{2} \alpha / \partial \xi_{P}^{2}, \partial^{2} \phi_{P} / \partial \xi_{P}^{2}$ and the product of first derivatives $\partial \alpha / \partial \xi_{P}$ and $\partial \phi_{P} / \partial \xi_{P}$ are $O\left(R_{0}^{-2}\right)$, and hence negligible if only terms of $O\left(R_{0}^{-1}\right)$ and larger are retained in the analysis.

Therefore, retaining only terms of $O\left(R_{0}^{-1}\right)$ and larger leads to the derivation of the linear $\mathrm{PSE}$, which written in operator form is

$$
\left(L_{0}+L_{1}+L_{2}\right) \phi_{P}+M_{1} \frac{\partial \phi_{P}}{\partial \xi_{P}}+\frac{d \alpha}{d \xi_{P}} M_{2} \phi_{P}=0,
$$

where $L_{0}, L_{1}, L_{2}, M_{1}$ and $M_{2}$ are differential operators which depend on $R_{0}, \xi_{P}, \alpha, \Psi_{B}$ and $U_{f}$ (see (30) for their full form). The operator $L_{0}$ is the parallel Orr-Sommerfeld operator, $L_{1}$ accounts for the transverse velocity component of the mean flow, and $L_{2}$ incorporates the coordinate transformation (2.3).

In the partition (2.4) there is ambiguity in the choice of the functions $\alpha\left(\xi_{P}\right)$ and $\phi_{P}\left(\xi_{P}, N_{P}\right)$, which is resolved by the introduction of the normalization condition

$$
\int_{0}^{\infty} \frac{\partial \phi_{P}}{\partial \xi_{P}} \phi_{P}^{\dagger} d N_{P}=0,
$$

where $\nmid$ denotes the complex conjugate. This normalization condition minimizes the 
streamwise change $\partial \phi_{P} / \partial \xi_{P}$ in a weighted sense over the $N_{P}$-domain, which also keeps $\partial \phi_{P} / \partial \xi_{P}$ small in accordance with the initial assumption. Equations (2.5) and (2.6) are solved numerically via a spectral collocation technique using Chebyshev polynomials. This method is equivalent to that described in (24) and (19), which the reader is directed to for more details of the scheme.

In this study we are only interested in the propagation of the eigenmodes, generated in the leading-edge region of the body, through the Orr-Sommerfeld region. Therefore (2.5) is solved with homogeneous boundary conditions, and an upstream boundary condition stipulated by

$$
\phi_{P}\left(\xi_{0}, N_{P}\right)=\Phi\left(\xi_{0}, N_{P}\right), \quad \alpha\left(\xi_{0}\right)=\alpha_{0},
$$

where $\xi_{0}$ is the dimensionless starting position for the analysis along the surface of the body. This boundary condition depends upon the form of the boundary layer at $\xi_{0}$ and the interaction of the free-stream disturbance with the boundary layer upstream of this point.

This paper uses the boundary condition given by (30) which is the first Lam-Rott eigenmode of the asymptotic receptivity analysis in the vicinity of the leading edge $(\mathbf{1 2}, \mathbf{1 7}, \mathbf{3 0})$. This mode is chosen because it is the only discrete eigenmode which exhibits streamwise growth after the lower branch neutral stability point and hence will dominate the solution far downstream. The advantage of this receptivity condition, as apposed to other upstream boundary conditions, is that it contains all the information required to give the amplitude of the unstable $\mathrm{T}-\mathrm{S}$ wave as it enters the Orr-Sommerfeld region. Hence, the complete $\mathrm{T}-\mathrm{S}$ wave amplitude downstream is known. This is discussed in more detail in (19) for the case of a flat plate. This boundary condition has the form

$$
\Phi=\psi_{1}^{\mathrm{LR}}=C_{1}\left(\sigma R_{0} \xi_{P}\right)^{\tau_{1}} g_{0}\left(\xi_{P}, N_{P}\right)
$$

where $C_{1}$ is the receptivity coefficient $(\mathbf{1 5}, \mathbf{1 6}, \mathbf{1 7})$ and $\tau_{1}=-0.6921-7.9508 \gamma_{1} i(\mathbf{1 7}, \mathbf{2 9})$. The quantity $\sigma$ is represented by $R e^{-1}=\epsilon^{6}$ in $(\mathbf{1 9})$ and (30). The mode shape $g_{0}$ takes the form

$$
\begin{aligned}
g_{0}\left(\xi_{P}, N_{P}\right) & =\left(\left(2 \sigma R_{0} \xi_{P}\right)^{1 / 2} f^{\prime}\left(N_{P}\right)+\lambda \frac{\int_{0}^{M}(M-\widetilde{M}) A i(\tilde{z}) d \widetilde{M}}{\int_{0}^{\infty} \operatorname{Ai}(\tilde{z}) d \widetilde{M}}-\lambda M\right) \\
& \times \exp \left(-\frac{\sigma^{3 / 2} R_{0} \sqrt{ } 2(1+i) \xi_{P} N_{P}}{\lambda \hat{\gamma}\left(\xi_{P}\right) \rho_{1}^{3 / 2}}\right),
\end{aligned}
$$

where

$$
\begin{aligned}
M & =\left(2 \sigma R_{0} \xi_{P}\right)^{1 / 2}\left(1-\frac{\gamma_{1}}{\sigma R_{0} \xi_{P}}\right) N_{P} \\
\tilde{z} & =-\rho_{1}+\rho_{1}^{-1 / 2} e^{i \pi / 4} \widetilde{M} \\
\hat{\gamma} & =1-1.2023 \gamma_{1} \frac{\ln \left(\sigma R_{0} \xi_{P}\right)}{\sigma R_{0} \xi_{P}}+\left(D+3 \gamma_{1}\right) \frac{1}{\sigma R_{0} \xi_{P}}+O\left(\xi_{P}^{-1.887}\right)
\end{aligned}
$$

The function $f$ is the usual Blasius function which satisfies

$$
f^{\prime \prime \prime}+f f^{\prime \prime}=0, \quad f(0)=f^{\prime}(0)=0, \quad f^{\prime} \longrightarrow 1 \quad \text { as } \quad N_{P} \longrightarrow \infty,
$$




\begin{tabular}{ccc}
\hline & Flat Plate & Parabola \\
\hline$U_{f}$ & 1 & $\frac{\left(2 \sigma R_{0} \xi_{P}\right)^{1 / 2}}{\left(2 \sigma R_{0} \xi_{P}+S\right)^{1 / 2}}$ \\
$\gamma_{1}$ & 0 & $-S / 4$ \\
$D$ & 0 & $\frac{S}{2}\left(2.075-0.60115 \ln \left(\frac{S}{2}\right)\right)$ \\
\hline
\end{tabular}

Table 1 Values of the parameters used in the upstream PSE boundary condition for both the semi-infinite flat plate and the parabolic body.

where the primes denote $d / d N_{P}$. The constant $\lambda=f^{\prime \prime}(0)=0.4696 \ldots$, while the constant $D$ is calculated numerically and depends upon the curvature of the body $(\mathbf{1 6}, \mathbf{1 7})$. The function $E\left(N_{P}\right)$ is also calculated numerically by solving the differential equation

$$
E^{\prime \prime \prime}+f E^{\prime \prime}+2 f^{\prime} E^{\prime}-f^{\prime} E=-2\left(f^{\prime}-1\right)-2.4046 f f^{\prime \prime} .
$$

The constant $\rho_{1}=1.0188 \ldots$ is the first root of $A i^{\prime}\left(-\rho_{i}\right)=0$ where $A i$ is the Airy function of the first kind, and $\gamma_{1}$ depends upon the form of the slip velocity $U_{f}$.

The initial value for the wavenumber $\alpha$ is given by

$$
\alpha\left(\xi_{0}\right)=\frac{i \sigma^{5 / 3} R_{0} e^{-i \pi / 4} \sqrt{2} \xi_{0}}{\rho_{1}^{3 / 2} \lambda}\left(1+1.2023 \gamma_{1} \frac{\ln \left(\xi_{0}\right)}{\xi_{0}}-\left(D+3 \gamma_{1}\right) \frac{1}{\xi_{0}}\right)
$$

and the form of the base flow is

$\Psi_{B}=\frac{\left(2 \xi_{P}\right)^{1 / 2}}{R_{0}^{1 / 2}}\left(f-1.2023 \gamma_{1}\left(N_{P} f_{N_{P}}-f\right) \frac{\ln \left(\sigma R_{0} \xi_{P}\right)}{\sigma R_{0} \xi_{P}}+\frac{D\left(N_{P} f_{N_{P}}-f\right)+\gamma_{1} E\left(N_{P}\right)}{\sigma R_{0} \xi_{P}}\right)+O\left(\xi_{P}^{-1.387}\right)$.

The current study investigates the propagation of the $\mathrm{T}-\mathrm{S}$ wave on a semi-infinite flat plate and a parabolic body. The values of $U_{f}, \gamma_{1}$ and $D$ for each body are given in table 1 , where $S$ is the Strouhal number defined by $S=r_{n} \omega^{*} / U_{\infty}$ and $r_{n}$ is the dimensional nose radius of the body, see $(\mathbf{3 0})$ for more details.

\subsection{Formulation of the DNS scheme}

The DNS approach used to compare to the PSE results of $\S 2.1$ is the scheme investigated in (18). This scheme is chosen because it is computationally faster than schemes which solve the full unsteady Navier-Stokes equations. A summary of the formulation is presented here, and the reader is directed to $(\mathbf{1 8})$ for full details of the formulation and the numerical method.

The non-dimensional Navier-Stokes equations in stream function $(\psi)$ and vorticity $(\Omega)$ variables, written in terms of parabolic coordinates $(\xi, N)$, are given by

$$
\begin{aligned}
\frac{\partial^{2} \psi}{\partial \xi^{2}}+\frac{\partial^{2} \psi}{\partial N^{2}}-\left(\xi^{2}+N^{2}\right) \Omega & =0 \\
\left(\frac{\partial}{\partial \xi^{2}}+\frac{\partial}{\partial N^{2}}+\frac{\partial \psi}{\partial \xi} \frac{\partial}{\partial N}-\frac{\partial \psi}{\partial N} \frac{\partial}{\partial \xi}-\left(\xi^{2}+N^{2}\right) \frac{\partial}{\partial t}\right) \Omega & =0
\end{aligned}
$$


The parabolic coordinate system $(\xi, N)$ is related to the Cartesian $(x, y)$ coordinates via

$$
x=\frac{1}{2}\left(\xi^{2}-N^{2}\right), \quad y=\xi N,
$$

where all the variables have been non-dimensionalised by the length scale $\nu / U_{\infty}$ and the time scale $\nu / U_{\infty}^{2}$. Note that $\xi \in[0, \infty]$ denotes the upper surface of the body and $\xi \in[0,-\infty]$ denotes the lower surface, with the leading edge of the body at $\xi=0$. The non-dimensional nose radius of the parabola is given by $R=r_{n} U_{\infty} / \nu$, and in the limit of an infinitely thin flat plate $(R \rightarrow 0)$ equations (2.12) and (2.13) are singular. This singularity is removed by introducing the new variables $\tilde{f}$ and $\tilde{g}$, as suggested by Davis (41), which are related to $\psi$ and $\Omega$ by

$$
\psi=\xi \tilde{f}(\xi, N, t), \quad \Omega=-\frac{\xi}{\left(\xi^{2}+N^{2}\right)} \tilde{g}(\xi, N, t) .
$$

This will allow us to investigate the receptivity results on a semi-infinite flat plate as well as a parabolic body. These new variables are substituted into (2.12) and (2.13) to give equations (10) and (11) of (18). These equations are solved for the variables $\tilde{f}$ and $\tilde{g}$ with the following five boundary conditions. The no-slip and no-penetration conditions give

$$
\tilde{f}=\frac{\partial \tilde{f}}{\partial N}=0
$$

along the surface of the parabola $N=R^{1 / 2}$. The vorticity at the wall is given by

$$
\tilde{g}\left(\xi, R^{1 / 2}\right)=\frac{\partial^{2} \tilde{f}}{\partial N^{2}},
$$

while at the free-stream

$$
\frac{\partial \tilde{f}}{\partial N} \rightarrow 1 \quad \text { and } \quad \tilde{g} \rightarrow 0 \quad \text { as } \quad N \rightarrow \infty
$$

The receptivity response of these equations to an incoming acoustic disturbance can now be investigated.

\subsubsection{Receptivity to an acoustic disturbance}

As in (2.4) for the formulation of the PSE, we look for a solution to the system of equations (10) and (11) of (18) when the free-stream velocity is decomposed into a sum of a steady uniform flow parallel to the surface of the body and a small perturbation of frequency $\sigma=\nu \omega^{*} / U_{\infty}^{2}$. Here $\omega^{*}$ is the dimensional frequency of the acoustic disturbance. Thus

$$
\left.\psi\right|_{N \rightarrow \infty}=\left(1+\delta e^{-i \sigma t}\right) \xi N
$$

where $\delta \ll 1$ is the amplitude of the disturbance, which is sufficiently small for linearization. Note here, that this form of acoustic disturbance differers from equation (16) of (18) by the addition of a minus sign in the exponential. This is so that direct comparisons can be made with the PSE results, otherwise the two methods would be complex conjugates of one another. 
The variables $\tilde{f}$ and $\tilde{g}$ are decomposed into a steady basic flow and an unsteady perturbation flow by introducing functions the form

$$
\begin{aligned}
& \tilde{f}(\xi, N, t)=F(\xi, N)+\delta e^{-i \sigma t} f(\xi, N), \\
& \tilde{g}(\xi, N, t)=G(\xi, N)+\delta e^{-i \sigma t} g(\xi, N) .
\end{aligned}
$$

Substituting these expressions into both the governing equations and the boundary conditions (equations (10) and (11) of (18) and (2.14)-(2.16) here) and equating powers of $\delta$, gives a system of two equations at each order of $\delta$ governing both the steady base flow and the unsteady perturbation flow.

At $O\left(\delta^{0}\right)$ we obtain the steady flow equations

$$
\begin{aligned}
& F_{N N}-G+F_{\xi \xi}+\frac{2}{\xi} F_{\xi}=0 \\
& G_{N N}+\left(F+\xi F_{\xi}-\frac{4 N}{\xi^{2}+N^{2}}\right) G_{N} \\
& +\left(\frac{\xi^{2}-N^{2}}{\xi^{2}+N^{2}} F_{N}-\frac{2 N}{\xi^{2}+N^{2}} F-\frac{2 \xi N}{\xi^{2}+N^{2}} F_{\xi}\right) G \\
& -\left(\xi F_{N}+\frac{4 \xi}{\xi^{2}+N^{2}}\right) G_{\xi}+G_{\xi \xi}+\frac{2}{\xi} G_{\xi}=0
\end{aligned}
$$

with the boundary conditions

$$
\begin{array}{r}
F=0, \quad F_{N}=0 \quad \text { and } \quad G=F_{N N} \quad \text { on } \quad N=R^{1 / 2} \\
F_{N} \rightarrow 1 \quad \text { and } \quad G \rightarrow 0 \quad \text { as } \quad N \rightarrow \infty .
\end{array}
$$

These base flow equations are steady, coupled and nonlinear in the variables $F$ and $G$. The downstream boundary conditions as $\xi \rightarrow \pm \infty$ ( $+\infty$ for upper surface, $-\infty$ for lower surface) are such that the disturbances pass through this boundary without causing reflections which significantly alter the flow upstream. This is achieved numerically by suppressing the elliptic terms (final two terms) of each governing equation using a buffer domain $(\mathbf{1 8}, \mathbf{4 2})$.

At $O(\delta)$ we obtain the equations for the unsteady perturbation flow

$$
\begin{aligned}
& f_{N N}-g+f_{\xi \xi}+\frac{2}{\xi} f_{\xi}=0 \\
& g_{N N}+\left(F+\xi F_{\xi}-\frac{4 N}{\xi^{2}+N^{2}}\right) g_{N}-\left(\xi F_{N}+\frac{4 \xi}{\xi^{2}+N^{2}}\right) g_{\xi} \\
& +\left(\frac{\xi^{2}-N^{2}}{\xi^{2}+N^{2}} F_{N}-\frac{2 N}{\xi^{2}+N^{2}} F-\frac{2 \xi N}{\xi^{2}+N^{2}} F_{\xi}\right) g+\left(\frac{\xi^{2}-N^{2}}{\xi^{2}+N^{2}} G-\xi G_{\xi}\right) f_{N} \\
& +\left(\xi G_{N}-\frac{2 \xi N}{\xi^{2}+N^{2}} G\right) f_{\xi}+\left(G_{N}-\frac{2 N}{\xi^{2}+N^{2}} G\right) f \\
& +i \sigma\left(\xi^{2}+N^{2}\right) g+g_{\xi \xi}+\frac{2}{\xi} g_{\xi}=0,
\end{aligned}
$$

with the boundary conditions

$$
\begin{array}{r}
f=0, \quad f_{N}=0 \quad \text { and } \quad g=f_{N N} \quad \text { on } \quad N=R^{1 / 2}, \\
f_{N} \rightarrow 1 \quad \text { and } \quad g \rightarrow 0 \text { as } \quad N \rightarrow \infty .
\end{array}
$$


These perturbation flow equations are steady, coupled and linear in the variables $f$ and $g$, and their coefficients depend upon the base flow. These equations also have the downstream boundary condition that the disturbance must pass through the boundary as $\xi \rightarrow \pm \infty$ without causing reflections. This is again resolved by using a buffer domain.

The base flow equations are solved via an iterative scheme, where they are first linearized using Newton's linearization technique, where for example, the $n^{\text {th }}$ iteration of the product $F G$ is written as

$$
F^{(n)} G^{(n)}=F^{(n)} G^{(n-1)}+F^{(n-1)} G^{(n)}-F^{(n-1)} G^{(n-1)} .
$$

The resulting system of linear algebraic equations are solved using an iterative scheme until the absolute difference between successive iterations of $F$ and $G$ becomes less than $10^{-6}$. These functions then enter the coefficients for $f$ and $g$ which are solved simultaneously using a standard banded matrix solver. For full details of the numerical scheme to solve equations (2.19) $-(2.26)$ the reader is referred to $(\mathbf{1 8})$ and $(\mathbf{4 2})$.

\subsubsection{Numerical solution of DNS method}

Both the basic flow equations and the perturbation equations are solved on a rectangular grid of size $-\xi_{\max } \leqslant \xi \leqslant \xi_{\max }$ and $0 \leqslant N-R^{1 / 2} \leqslant N_{\max }$. The grid is discretized into $2 Z-1$ points in the $\xi$-direction and $Y$ points in the $N$-direction. As we are only considering a basic flow which is perpendicular to the nose of the body, we need only consider the upper surface of the parabola from $0 \leqslant \xi \leqslant \xi_{\max }$. The grid points are concentrated close to regions of large velocity gradients, near the wall and close to the leading edge, by using Robert's stretching transformation (43). This transformation takes the form

$$
\xi=\xi_{\max } \frac{\left(\beta_{\xi}+1\right)-\left(\beta_{\xi}-1\right)\left(\left(\beta_{\xi}+1\right) /\left(\beta_{\xi}-1\right)\right)^{1-\bar{\xi}}}{\left(\left(\beta_{\xi}+1\right) /\left(\beta_{\xi}-1\right)\right)^{1-\bar{\xi}}+1},
$$

where $\bar{\xi}$ are uniform grid points in the range $[0,1]$ and $\beta_{\xi}$ is the stretching parameter in the $\xi$-direction. This study uses $\beta_{\xi}=1.25$ and $\beta_{N}=1.005$, along with $\xi_{\max }=1.5 \times 10^{6}$ and $N_{\max }=35$. The value of $\xi_{\max }$ is large enough to capture the evolution of $\mathrm{T}-\mathrm{S}$ waves down to $\sigma=60 \times 10^{-6}$, and the value of $N_{\max }$ is approximately 10 times thicker than the maximum boundary layer thickness. The value of $\xi$, and hence $\xi_{\max }$, can be converted into the streamwise Reynolds number, $R e_{x}$, by

$$
R e_{x}=\frac{U_{\infty} x^{*}}{\nu}=\frac{1}{2} \xi^{2}-R .
$$

The neutral stability curve for the flat plate and parabola, calculated via the PSE, showing the computational domain is given in figure 1.

To examine the sensitivity of the numerical results to the number of computational grid points, some particular measure of the flow has to be compared as the number of grid points is varied. Haddad \& Corke (18) chose to do this by comparing the function $G\left(\xi, N=R^{1 / 2}\right)$. However, although two different grid sizes may give graphically identical $G\left(\xi, N=R^{1 / 2}\right)$, they may not give the same level of agreement for the perturbation quantities. Therefore, figure 2 plots the absolute value of the wall vorticity on a flat plate $|g / \xi|$ for $\hat{\sigma}=\sigma \times 10^{6}=100$ as a function of $\xi$ for various grid sizes. In panel (a) we fix the value of $Z=2000$ and vary 


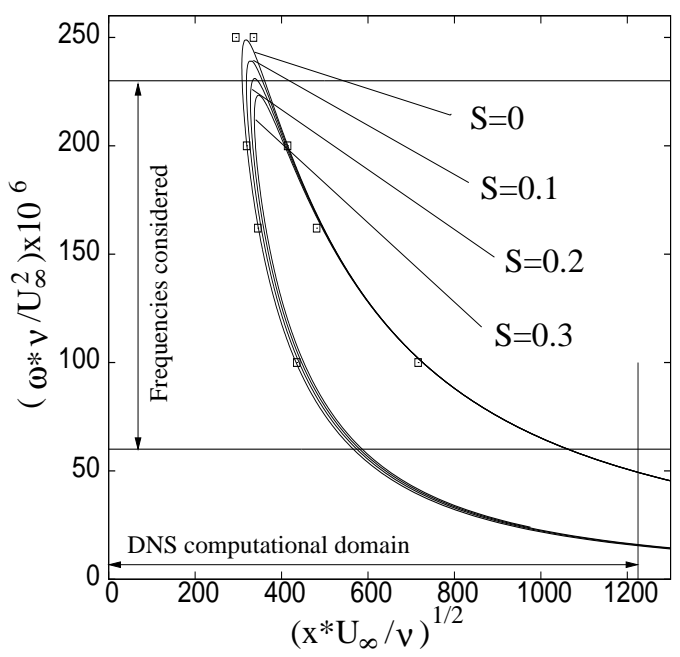

Fig. 1 Plot of the neutral stability curve, calculated via the PSE, for the flat plate and parabola with $S=0.1,0.2$ and 0.3 . The squares show the experimental data of Klingmann et al. (44)
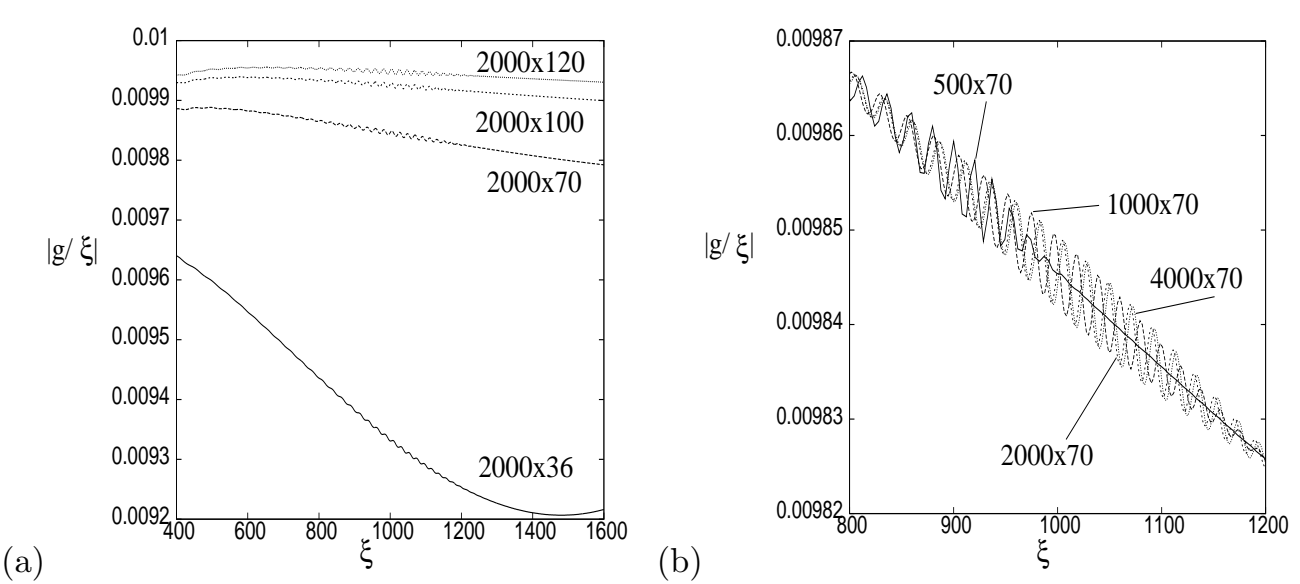

Fig. 2 Plot of the wall vorticity $|g / \xi|$ on a flat plate with $\hat{\sigma}=100$, showing the effect of varying the number of grid points $Z \times Y$. 
$Y$, the number of grid points in the $N$-direction, while in panel (b) we fix $Y=70$ and vary $Z$. For each of the grid sizes in this figure, the basic flow variables $F$ and $G$ are graphically identical, but clearly there are subtle differences in the perturbation quantities. Therefore, to be able to extract the correct $\mathrm{T}-\mathrm{S}$ wave information, these perturbation quantities need to be grid invariant. In panel (b) it is seen that increasing the value of $Z$ for a fixed value of $Y$ does not change the magnitude of the oscillations observed, only their wavelength and the streamwise distance over which they are observed. In panel (a) it appears that varying $Y$ for a fixed value of $Z$ makes a significant difference to the wall vorticity, however once the $\mathrm{T}-\mathrm{S}$ wave information has been filtered out from these perturbation variables (see later in this section) the results with $Y=100$ and 120 are almost identical (see figure 6). Therefore, in this paper a grid size of $Z \times Y=2000 \times 100$ is used, as this produces results which are practically grid independent, but the simulations do not use up all the available computer memory, which greatly increases the computation time.

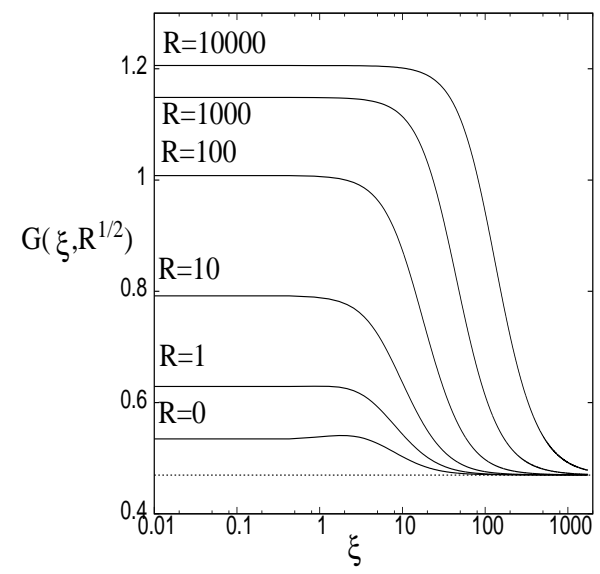

Fig. 3 Plot of the skin friction coefficient, $G\left(\xi, R^{1 / 2}\right)$ on a parabolic body for various values of $R$. The dotted line represents the result for the Blasius boundary layer.

Figure 3 plots the skin friction coefficient $G\left(\xi, R^{1 / 2}\right)$ for various values of $R$. These results are in agreement with those of $(\mathbf{1 8})$ and $(\mathbf{3 5})$. The horizontal dotted line is the value $\lambda=0.4696$ which is the skin friction coefficient for the Blasius boundary layer, and this figure shows that all the results tend to this solution far downstream, which is a good check on the numerical scheme.

The results for the perturbation quantities $f$ and $g$ consist of a Stokes wave, determined by the local boundary layer forcing at that location, a sum of discrete T-S modes, one of which exhibits growth in the streamwise direction and is of interest to us in this study, and non-modal eigenmodes of the continuous spectrum $(\mathbf{2 0})$. In order to extract the growing $\mathrm{T}-$ $\mathrm{S}$ wave the Stokes wave is subtracted off using the same method as (18). This method solves (2.23)-(2.26) to find both $f$ and $g$, and then these equations are solved again with $F=G=0$ to obtain the local Stokes wave solution. This is then subtracted from the previous result to give the $\mathrm{T}-\mathrm{S}$ wave evolution. However, as suggested by (19), this calculation does not 
remove the whole contribution of the Stokes wave and a remnant is left behind that needs to be dealt with. Hammerton (45) showed that the large- $\xi$ asymptotic form of the Stokes layer at the edge of the boundary layer can be written as

$\psi^{\text {Stokes }} \sim \xi\left(N-\frac{1+i}{(2 \sigma)^{1 / 2}} \xi^{-1}+\frac{i \beta}{\sigma} \xi^{-2}+\frac{13 \lambda}{8 \sigma^{2}} \xi^{-4}-\frac{39 \lambda i}{8 \sigma^{3}} \xi^{-6}-\frac{4051 \sqrt{2}(1-i) \lambda^{2}}{256 \sigma^{7 / 2}} \xi^{-7}+o\left(\xi^{-7}\right)\right)$

where $\lambda=f^{\prime \prime}(0) \approx 0.4696$ and $\beta=\lim _{N \rightarrow 0}\left(N f^{\prime}-f\right) \approx 1.217$. However, the method of solving (2.23)-(2.26) with $F=G=0$ will only give the leading order term

$$
\psi^{\text {Stokes }} \sim \xi N
$$

see (46). The remnant left in the DNS scheme can be seen in figure 4 which plots $\left.(f(\xi, N)-N)\right|_{N=3.07}$ for (a) $\hat{\sigma}=100$ and (b) $\hat{\sigma}=200$. The value $N=3.07$ is chosen

(a)

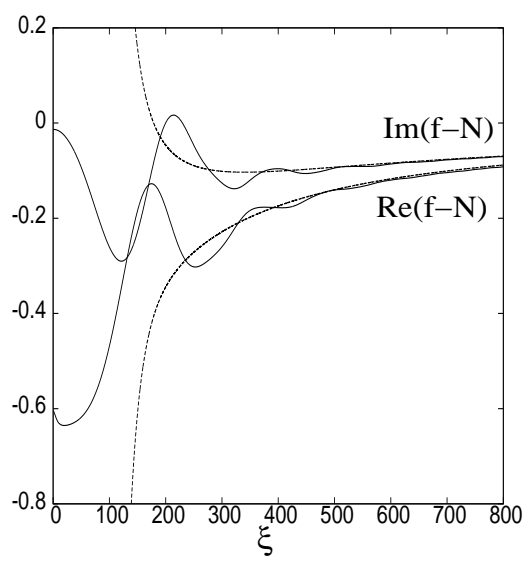

(b)

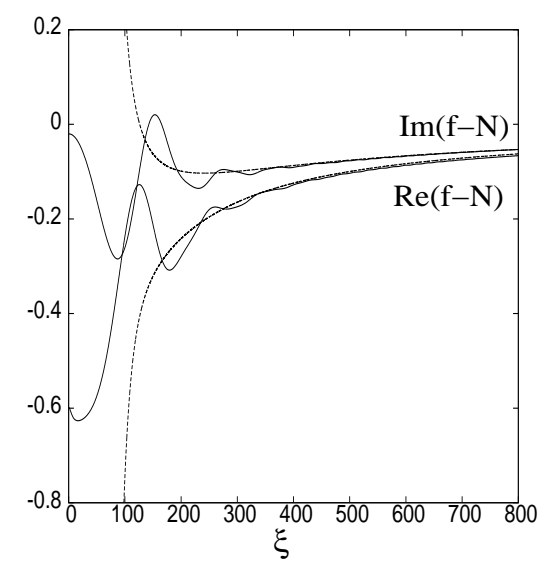

Fig. 4 Plot of real and imaginary parts of $\left.(f(\xi, N)-N)\right|_{N=3.07}$ (solid lines) at the edge of the boundary layer for a flat plate, $R=0$, and the large- $\xi$ asymptotic form given by $(2.27) / \xi-N$ (dashed lines) for (a) $\hat{\sigma}=100$ and (b) $\hat{\sigma}=200$.

because it is a point approximately at the edge of the boundary layer. In this figure it can be seen that the DNS numerical result (solid lines) agrees well with the asymptotic result from (2.27) (dashed lines) at large $\xi$. This solution also contains a contribution from the non-modal eigenmodes of the continuous spectrum.

The Stokes layer remnant and the non-modal eigenmodes are removed by 'filtering' out the growing $\mathrm{T}-\mathrm{S}$ wave in the manner described in (18). A one-dimensional Gaussian filter is applied to the Stokes-layer-removed perturbation results in the $\xi$-direction for each value of $N$. The filter is designed to suppress any disturbances which have wavelengths that are greater than or equal to twice the expected discrete $\mathrm{T}-\mathrm{S}$ wave wavelength. This wavelength is chosen so as it is assumed that the eigenmodes from the continuous spectrum have wavelengths greater than twice the discrete $\mathrm{T}-\mathrm{S}$ wave wavelength (18). An example 
(a)

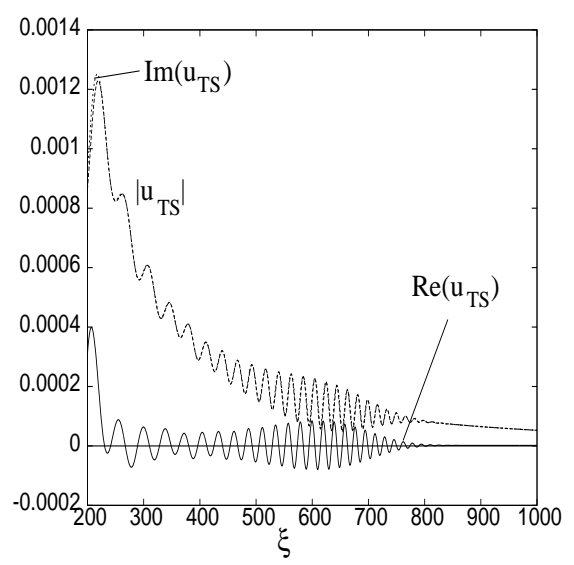

(b)

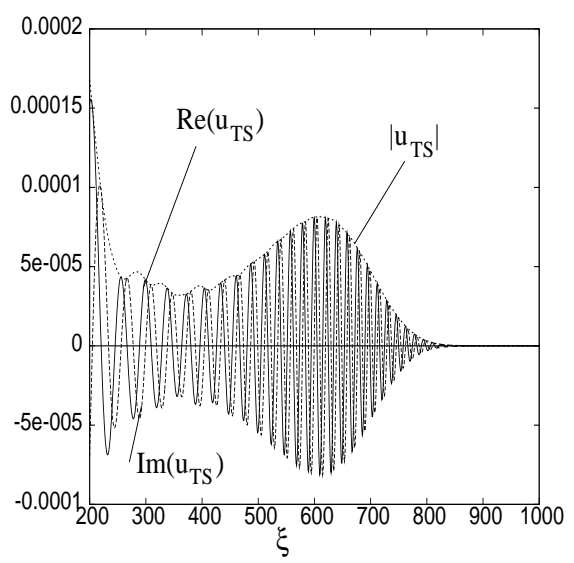

Fig. 5 Plot of the real and imaginary parts of $u_{\mathrm{TS}}(\xi, N=0.035)$ on a flat plate with $\hat{\sigma}=200$ where (a) the result is not filtered, and (b) where it has been filtered. In panel (a), $\operatorname{Im}\left(u_{\mathrm{TS}}\right)$ and $\left|u_{\mathrm{TS}}\right|$ are almost indistinguishable from one another.

of this filtering process can be seen in figure 5, which plots the real and imaginary parts of the T-S wave velocity, $u_{\mathrm{TS}}$, (a) before and (b) after filtering at $N=0.035$ for a flat plate with $\hat{\sigma}=200$. In panel (a) $\operatorname{Re}\left(u_{\mathrm{TS}}\right)$ is centred on the $\xi$-axis as expected, but $\operatorname{Im}\left(u_{\mathrm{TS}}\right)$ clearly shows a different behaviour as it too should be centred on the $\xi$-axis if it were solely comprised of the T-S wave. This remnant has to be removed because it affects the results by dominating the $\left|u_{\mathrm{TS}}\right|$ result. Once the Gaussian filter has been applied to the result, the $\operatorname{Im}\left(u_{\mathrm{TS}}\right)$ result is bought down to the $\xi$-axis and now $\left|u_{\mathrm{TS}}\right|$ gives the envelope of the $\mathrm{T}-\mathrm{S}$ wave as we would expect. This makes calculating quantities such as the position of the upper branch point much easier, and comparisons with the PSE results, which have no Stokes layer or non-modal eigenmode contributions, will also be easier and cleaner.

In figure $6\left|u_{\mathrm{TS}}(\xi, N=0.073)\right|$ is plotted for a flat plate with $\hat{\sigma}=100$ for the different grid sizes $2000 \times 36,2000 \times 70,2000 \times 100$ and $2000 \times 120$ numbered $1-4$ respectively. This figure confirms that the difference seen between results 3 and 4 in figure 2(a) is greatly reduced after filtering, and so using a $2000 \times 100$ grid in this study will give consistent, grid independent results.

One final thing to note in this section is, the T-S wave amplitude $\left|u_{\mathrm{TS}}\right|$ in this paper is found by performing $\left|f_{N}-f_{N}^{\text {Stokes }}\right|$, as one might expect. However, in recreating the result in the lower panel of figure 13(b) of (18) we found that the value of $\left|u_{\mathrm{TS}}\right|$ in (18) can only be reproduced by performing $\left|f_{N}\right|-\left|f_{N}^{\text {Stokes }}\right|$, which does not actually give the T-S wave. Therefore, the claim made by (18) that they see T-S wave velocity profiles upstream of the lower branch point is questionable. In fact the investigation in $\S 3.1$ here shows that this is not the case, and what is actually observed in (18) is a combination of the Stokes wave remnant and non-modal eigenmodes not removed by the filtering process.

Now that we know that the DNS receptivity results are independent of the grid resolution we can go on to compare these results to the receptivity results of the PSE simulations outlined in $\S 2.1$. 


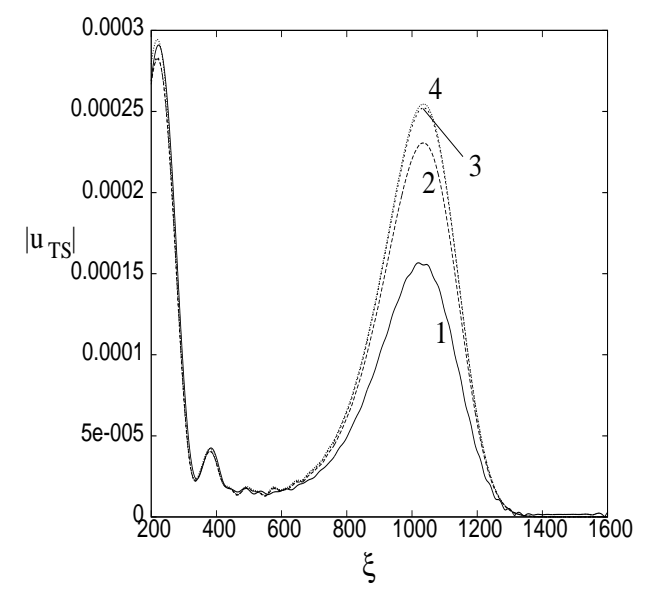

Fig. 6 Plot of $\left|u_{\mathrm{TS}}(\xi, N=0.073)\right|$ on a flat plate with $\hat{\sigma}=100$ showing the variation of changing the number of grid points in the $N$-direction. Here $Z=2000$ and $Y=36,70,100$ and 120 are numbered 1-4 respectively.

\section{Results}

In this section receptivity results are compared for the DNS and PSE on both a semi-infinite flat plate $(R=0)$ and a parabolic body $(R \neq 0)$ for the range of dimensionless acoustic frequencies $\hat{\sigma} \in[60,230]$. This range is chosen because, for the frequencies $\hat{\sigma}>230$ the DNS code produces less clean results, while for $\hat{\sigma}<60$ the upper branch neutral stability point for the DNS moves outside the range $\xi$ values that can be studied, see figure 1 . This range also covers the range of frequencies typically used in experiments (39). The small $\hat{\sigma}$ limit does not concern us in this study, because Turner (29) has already shown that in the small $\hat{\sigma}$ limit the growth rate and mode shape results of the PSE agreed well with the asymptotic results of Goldstein (2).

In this study we wish to calculate the position of the upper and lower branch neutral stability points for the T-S wave over all values of $N$. This means a growth rate must be considered which takes into account the peak T-S wave amplitude position varying with $N$ as well as $\xi$. For the DNS scheme this growth rate is simply given by

$$
G R(\xi)=\frac{\partial}{\partial \xi} \ln \left(u_{\mathrm{TS}}^{\max }\right)
$$

where $u_{\mathrm{TS}}(\xi, N)=u_{\mathrm{TS}}^{\max }(\xi) \bar{u}_{\mathrm{TS}}(\xi, N)$ where the maximum value of $\bar{u}_{\mathrm{TS}}$ is 1 . Using this same splitting of the amplitude function for the PSE gives

$$
\frac{\partial \phi_{P}}{\partial N_{P}}\left(\xi_{P}, N_{P}\right)=\left(\frac{\partial \phi_{P}}{\partial N_{P}}\right)^{\max }\left(\xi_{P}\right)\left(\overline{\frac{\partial \phi_{P}}{\partial N_{P}}}\right)\left(\xi_{P}, N_{P}\right),
$$


where the maximum value of $\left(\overline{\partial \phi_{P} / \partial N_{P}}\right)$ is 1 . Then $(2.4)$ shows that

$$
u_{\mathrm{TS}}=\frac{R_{0}^{1 / 2} U_{f}}{\left(2 \xi_{P}\right)^{1 / 2}} \frac{\partial \psi_{P}}{\partial N_{P}}=\left(\overline{\frac{\partial \phi_{P}}{\partial N_{P}}}\right)\left(\xi_{P}, N_{P}\right) \exp \left(\tilde{\theta}\left(\xi_{P}\right)-i \omega_{P} t_{P}\right)+\text { complex conjugate, }
$$

where $d \tilde{\theta} / d \xi_{P}=G R\left(\xi_{P}\right)$ is the $\mathrm{T}-\mathrm{S}$ wave growth rate which now contains all the wave amplitude information,

$$
G R(\xi)=\frac{\xi}{R_{0}}\left(i \alpha+\frac{\xi}{R_{0} U_{f}} \frac{1}{\left(\frac{\partial \phi_{P}}{\partial N_{P}}\right)^{\max }} \frac{\partial}{\partial \xi_{P}}\left(\frac{R_{0}^{1 / 2} U_{f}}{\left(2 \xi_{P}\right)^{1 / 2}}\left(\frac{\partial \phi_{P}}{\partial N_{P}}\right)^{\max }\right)\right) .
$$

The lower and upper branch neutral stability points are now defined as the positions where $\operatorname{Re}(G R)=0$.

In this study the amplitude of the unstable $\mathrm{T}-\mathrm{S}$ wave is required. This is achieved for the PSE method by integrating the growth rate $G R(\xi)$ from a position within the matching region between the leading edge and Orr-Sommerfeld region, $\xi^{\mathrm{LE}}$, to positions downstream. The value of $\xi^{\mathrm{LE}}$ can take any value in the region close the leading edge, where the downstream amplitude is independent of the choice of $\xi^{\mathrm{LE}}$. This region has been shown to exist for a flat plate by (19) and a parabolic body by (30). Thus, the amplitude of $u_{\mathrm{TS}}$ at the streamwise position $\xi$ can be written as

$$
u_{\mathrm{TS}}=\left.\frac{U_{f}\left(\xi^{\mathrm{LE}}\right)}{\sigma^{1 / 2} \xi^{\mathrm{LE}}} \frac{\partial \psi_{1}^{\mathrm{LR}}}{\partial N}\right|_{\xi^{\mathrm{LE}}} \exp \left(\int_{\xi^{\mathrm{LE}}}^{\xi} G R\left(\xi^{\prime}\right) d \xi^{\prime}\right) .
$$

The streamwise receptivity coefficient of (3.4) is defined as $K_{\xi}=\left|u_{\mathrm{TS}}\right|$ which agrees with the definition in (18), and this value at the lower (branch I) and upper (branch II) branch neutral stability points is denoted by $K_{I}$ and $K_{I I}$ respectively.

Turner \& Hammerton $(\mathbf{1 9}, \mathbf{3 0})$ showed that for small values of $\hat{\sigma}$, the PSE can be started back in the matching region, so the values of $G R(\xi)$ are well defined over the entire integration region in (3.4). However, for larger values of $\hat{\sigma}$ the PSE code cannot be initiated back in the matching region, and the growth rate has to be patched back to $\xi^{\mathrm{LE}}$ using the patching method described in (19). This patching method involves fixing the value of the growth rate and its derivative using both the leading edge solution and the PSE solution, and then patching the region in between using a cubic polynomial with complex coefficients. Turner (29) demonstrated that the T-S mode amplitude results using this patching technique agreed well with the asymptotic results of Goldstein (2).

\subsection{A semi-infinite flat plate}

This section focuses on the semi-infinite flat plate $(R=0)$. Quantities such as the position of the upper branch point, T-S wave mode shapes and $K_{\xi}$ are compared, for the two numerical methods. Here the receptivity coefficient $\left|C_{1}\right|=0.9662$.

Figure 7 plots the position of the upper branch neutral stability point, $\xi^{\mathrm{UB}}$ as a function of $\hat{\sigma}$. For the DNS results this point is calculated by plotting $u_{\mathrm{TS}}^{\max }$ for each value of $\xi$ and looking for the downstream turning point where $K_{\xi}$ has a local maximum. For small values of $\hat{\sigma}$ this turning point is well defined, but for larger values of $\hat{\sigma}$ this turning point 
(a)

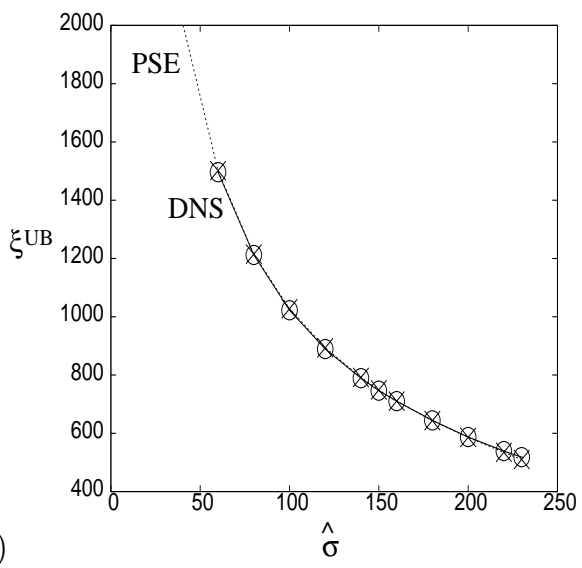

(b)

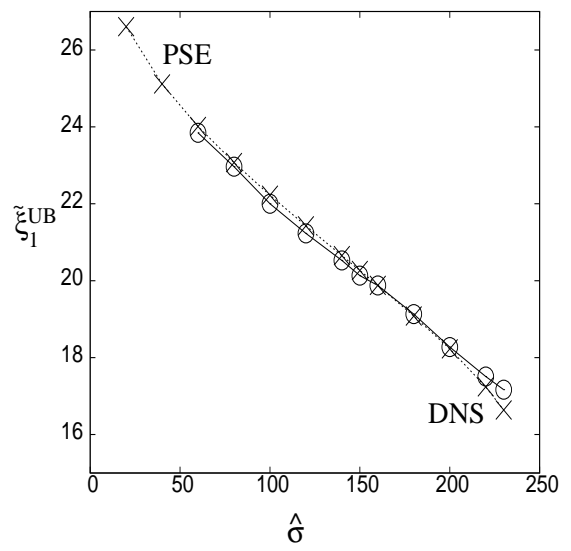

Fig. 7 Plot of the position of the upper branch neutral stability point as a function of $\hat{\sigma}$ for both the DNS (solid line, circles) and PSE results (dashed line, crosses). In panel (a) the results are plotted in the $\xi$ variable, while in panel (b) they are plotted in the $\tilde{\xi}_{1}=\xi^{2} \sigma^{4 / 3} / \lambda^{2}$ variable.

becomes obscured by oscillations which are superimposed on the result. In this case the position of the upper branch point is found by eye rather than numerically. The points in figure 7(a) show excellent agreement between the DNS (solid line, circles) and PSE (dashed line, crosses) results which is to be expected, as the modal T-S wave dominates the DNS solution at this point. In panel (b) these points are plotted using the streamwise variable $\tilde{\xi}_{1}=\xi^{2} \sigma^{4 / 3} / \lambda^{2}$ which scales with $\sigma$ and is used in (30). The main advantage of this variable is that it scales the lower branch neutral stability point to make it $O(1)$ which makes plotting the evolution of the $\mathrm{T}-\mathrm{S}$ wave amplitude clearer, as in figure 8. In panel (b) the agreement between these results appears to be less good than in panel (a), but the maximum percentage error between the two methods is just $3 \%$ for $\hat{\sigma}=230$ which is acceptable.

Figure 8 plots (a) $\left|u_{\mathrm{TS}}(\xi, N=0.035)\right|$ and (b) $\left|u_{\mathrm{TS}}\left(\tilde{\xi}_{1}, N=0.035\right)\right|$ for $\hat{\sigma}=$ 60, 100, 150, 200 and 230. The position of the upper branch points (PSE results denoted by the rightmost circles) agree well for both methods, but the DNS result gives a different position for the lower branch. For larger values of $\hat{\sigma}$ the lower branch point is less clear due to small oscillations in $\left|u_{\mathrm{TS}}\right|$. The difference observed between the two approaches is due to the Stokes layer remnant and the non-modal eigenmodes not being fully removed by the filtering process. Therefore, the DNS cannot directly give the T-S wave amplitude at the lower branch, although this value can be calculated via the $e^{N}$ method, such as in (7). The results in figure 8 are plotted at $N=0.035$, which is well inside the boundary layer, because at this value of $N$, the Stokes layer remnant and the continuous eigenmodes will only make a small contribution to the solution if not fully removed by the filtering process. This is because both these elements have mode shape peaks around $N \approx 2.5$ at the edge of the boundary layer $(\mathbf{2 0})$. Therefore, at larger values of $N$, such as at those where $K_{\xi}$ is calculated, there is a larger contribution of the Stokes layer remnant and nonmodal eigenmodes not removed by the filtering process. This means that either some of the 
(a)

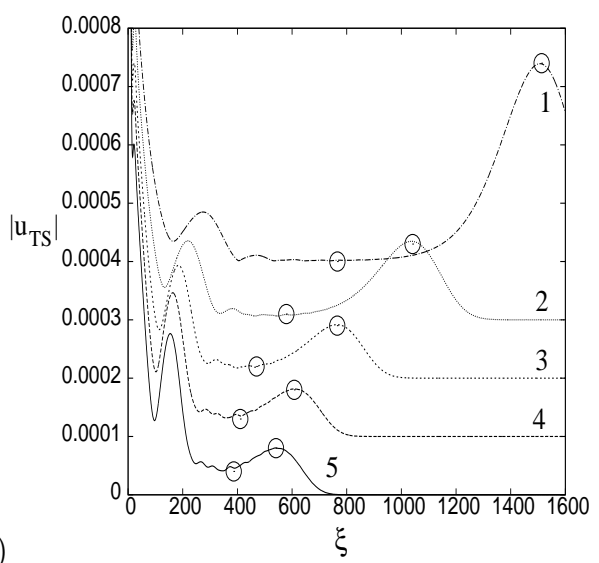

(b)

Fig. 8 Plot of (a) $\left|u_{\mathrm{TS}}(\xi, N=0.035)\right|$ and (b) $\left|u_{\mathrm{TS}}\left(\tilde{\xi}_{1}, N=0.035\right)\right|$ for $\hat{\sigma}=60,100,150,200$ and 230 numbered $1-5$ respectfully. The circles represent the positions of the lower and upper branch neutral stability points given by the PSE simulations. Here each result has been separated by the addition of the constant 0.0001 .

Stokes layer remnant still remains, or there are continuous, non-modal eigenmodes with wavelengths comparable to those of the T-S wave. This can be seen in figure 9 and in the mode shape plots in figure 10.

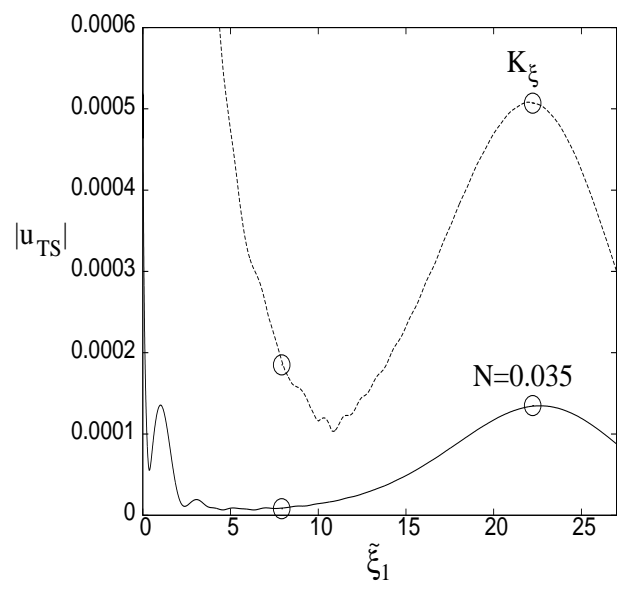

Fig. 9 Plot of $\left|u_{\mathrm{TS}}\right|$ as a function of $\tilde{\xi}_{1}$ for $\hat{\sigma}=100$ plotted at $N=0.035$ (result 2 from figure 8(b) with the added constant removed) and at the value of $N$ where the maximum value of $u_{\mathrm{TS}}$ occurs, this is $K_{\xi}$.

The mode shapes in figure 10 are normalized by their maximum value at that streamwise position, for both $\hat{\sigma}=100$ (left panels) and $\hat{\sigma}=200$ (right panels) for various streamwise 

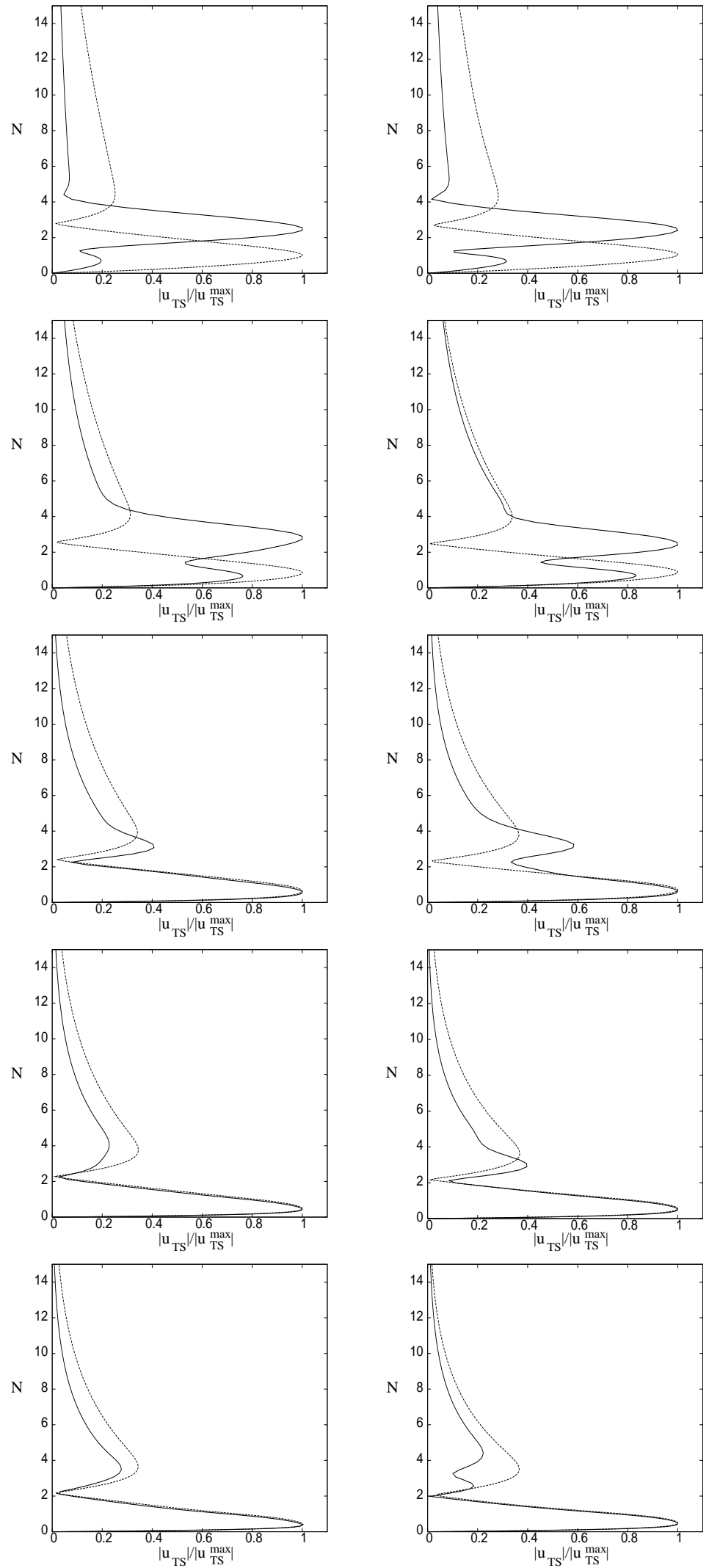

Fig. 10 Plot of the normalized T-S wave mode shape $\left|u_{\mathrm{TS}}\right| /\left|u_{\mathrm{TS}}^{\max }\right|$ for $\hat{\sigma}=100$ (left panels) and $\hat{\sigma}=200$ (right panels) for $\tilde{\xi}_{1}=5,10,15,20$ and 25 from top to bottom. In each panel the solid line gives the DNS result and the dashed line gives the PSE result. 
positions. In both cases, for the profiles at $\tilde{\xi}_{1}=5$, which is upstream of the lower branch point, the DNS profile (solid line) is dominated by a maximum at the outer edge of the boundary layer, and it is this peak which contaminates the $K_{\xi}$ result in figure 9 . The fact that the peak mode shape lies at the edge of the boundary layer suggests that it is either the Stokes layer remnant or a non-modal eigenmode. There is a secondary local maximum at a value of $N$ inside the boundary layer which approximately agrees with the position of the PSE maximum. If the mode shapes were renormalized with respect to this value, then the agreement between the profiles for $N \leqslant 2$ would be better. This confirms that the velocity profiles plotted in $(\mathbf{1 8})$, which are for streamwise values $\tilde{\xi}_{1}<1$, are not those of the T-S wave, even though their appearance suggests that they are. They are in fact a combination of T-S modes, non-modal eigenmodes and a Stokes layer remnant. Further downstream the maximum peak close to the wall begins to dominate the profile until at $\tilde{\xi}_{1}=15$ it agrees well with the maximum peak of the PSE result. Further downstream still, close to and beyond the upper branch point, the profiles remain in good agreement, except that the DNS profile slightly underestimates the PSE profile for $N>2$. If the real and

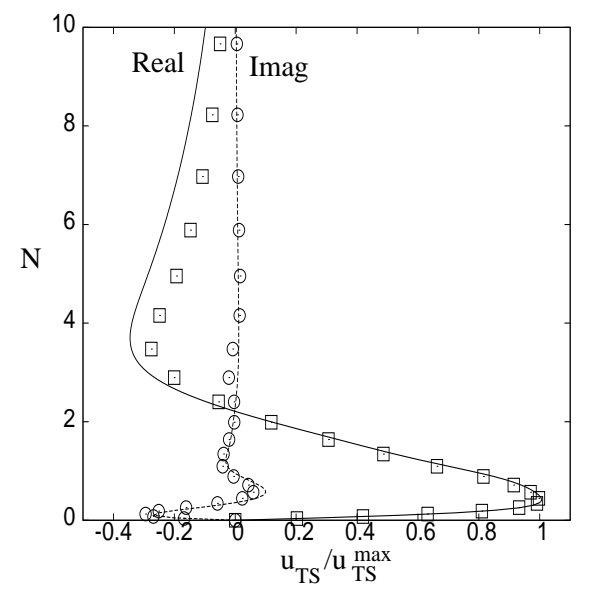

Fig. 11 Plot of the real and imaginary parts of the normalized T-S wave mode shape $u_{\mathrm{TS}} / u_{\mathrm{TS}}^{\max }$ at the upper branch point for $\hat{\sigma}=100$. The lines represent the PSE result while the squares and circles give the real and imaginary parts of the DNS result respectively.

imaginary parts of the mode shapes are explicitly compared in figure 11, we find that the imaginary part is very well approximated by the DNS solution, while the real part begins to differ for $N>2$ and thus gives the difference seen in figure 10 at large $\tilde{\xi}_{1}$.

To compare the values of $K_{\xi}$ from the two schemes, we must first examine the patching method outlined in (19). Figure 12 shows an example of the patching method for three different values of $\tilde{\xi}_{1}^{\mathrm{LE}}$. This figure demonstrates that the calculation of the growth rate patch changes as the value of $\tilde{\xi}_{1}^{\mathrm{LE}}$ varies, where $\tilde{\xi}_{1}^{\mathrm{LE}}$ is the point where the matching region with the leading edge result is assumed to occur. As $\tilde{\xi}_{1}^{\mathrm{LE}}$ is increased (from result 1 to result 3 ) the minimum value of $\operatorname{Re}(G R)$ decreases and this means the $\mathrm{T}-\mathrm{S}$ wave is damped more for result 3 than for result 1 , before it reaches the lower branch point. Therefore, as $\tilde{\xi}_{1}^{\mathrm{LE}}$ 


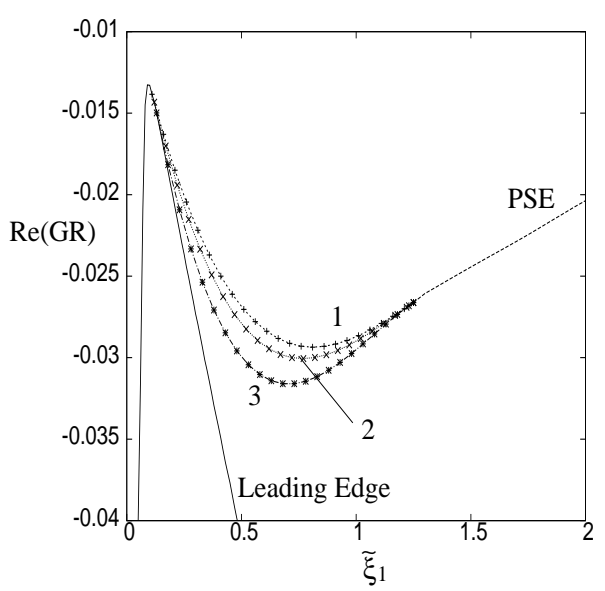

Fig. 12 Plot of $\operatorname{Re}(G R)$ for $\hat{\sigma}=100$ where the leading edge result from (2.10) and the PSE result are patched. The different patching results numbered $1-3$ are for $\tilde{\xi}_{1}^{\mathrm{LE}}=0.11,0.12$ and 0.13 respectively.

is increased, the value of $K_{I I}^{\mathrm{PSE}}$ will decrease until a value is reached where the quantity $\left|K_{I I}^{\mathrm{PSE}}-K_{I I}^{\mathrm{DNS}}\right|$ is minimised. At this point, we assume that the correct value of $\tilde{\xi}_{1}^{\mathrm{LE}}$ has been determined. This process can be used to determine $\tilde{\xi}_{1}^{\mathrm{LE}}$ because earlier in this section it was demonstrated that the T-S wave does dominate the DNS at the upper branch, so the two methods must agree at this point. The values of $\tilde{\xi}_{1}^{\mathrm{LE}}$ are plotted for various values of $\hat{\sigma}$ in figure 13. In the $\hat{\sigma} \rightarrow 0$ asymptotic limit, where the growth rate can be completely

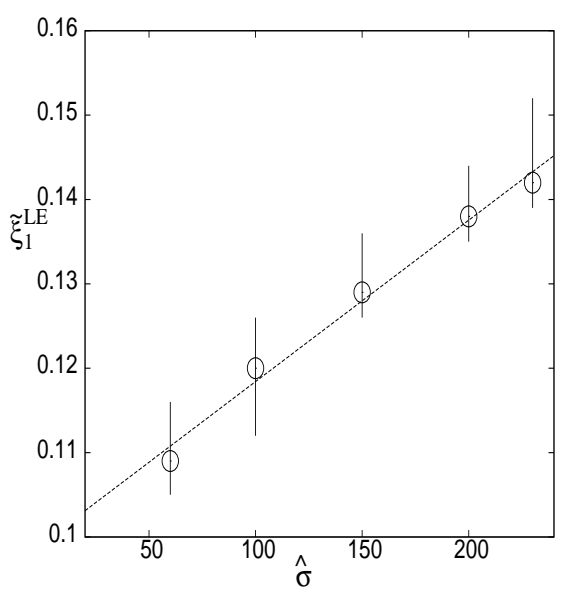

Fig. 13 Plot of $\tilde{\xi}_{1}^{\mathrm{LE}}(\hat{\sigma})$ showing the values for which $\left|K_{I I}^{\mathrm{PSE}}-K_{I I}^{\mathrm{DNS}}\right|$ is minimised. These are represented by the circles, while the solid lines correspond to the range of $\tilde{\xi}_{1}^{\mathrm{LE}}$ where $K_{I I}^{\mathrm{PSE}}$ is within $10 \%$ of $K_{I I}^{\mathrm{DNS}}$. The dashed line is the linear least squares fit of the data, $\tilde{\xi}_{1}^{\mathrm{LE}}=0.0993+0.000191 \hat{\sigma}$. 
determined back to the leading edge region, there is a range of $\tilde{\xi}_{1}$ values around $\tilde{\xi}_{1}^{\mathrm{LE}}$ which give a constant value of $K_{I I}$. This is the matching region. However, a consequence of having to patch the growth rate is, when the value of $\tilde{\xi}_{1}^{\mathrm{LE}}$ is varied, the patch itself and the value of $K_{I I}^{\mathrm{PSE}}$ also changes. Therefore, a range of $\tilde{\xi}_{1}$ values giving constant $K_{I I}^{\mathrm{PSE}}$ cannot be easily found. Therefore, error bars are plotted around the value of $\tilde{\xi}_{1}^{\mathrm{LE}}$ in figure 13, given by the solid lines, where the value of $K_{I I}^{\mathrm{PSE}}$ is within $10 \%$ of the value of $K_{I I}^{\mathrm{DNS}}$. By assuming a linear relation, such as the linear least squares fit marked on figure 13 , the value of $\tilde{\xi}_{1}^{\mathrm{LE}}$ for values of $\hat{\sigma}$ not considered in this study can also be found. This linear relation is not valid right back to $\hat{\sigma}=0$, as (19) have shown that when the growth rate is defined all the way back to the matching region, $\tilde{\xi}_{1}^{\mathrm{LE}} \approx 0.05$ for $\hat{\sigma}=0.016$. This linear relation is however, valid for the range of frequencies considered here.

(a)
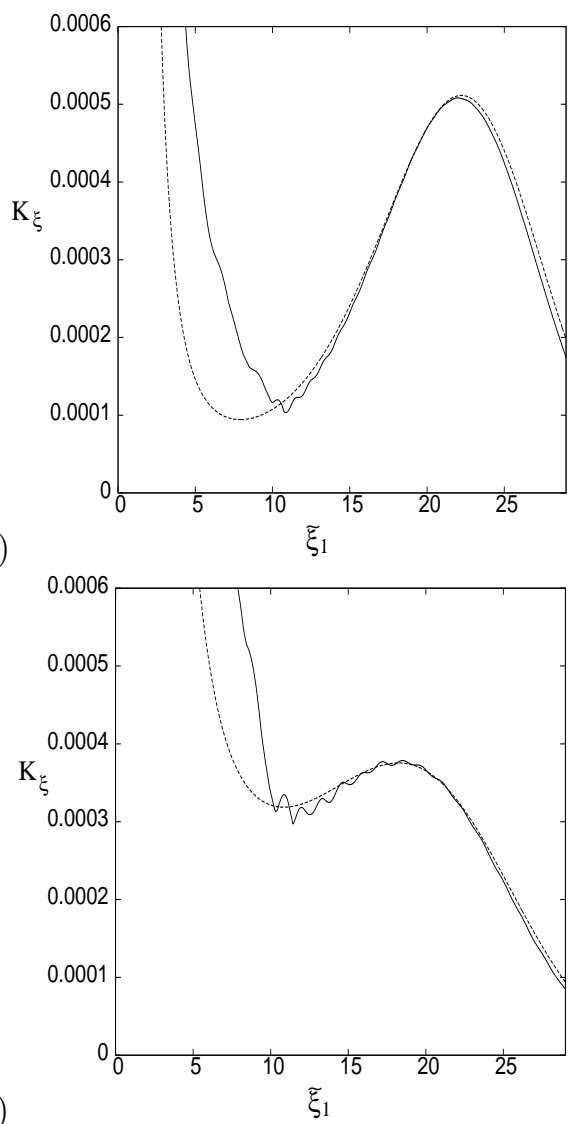

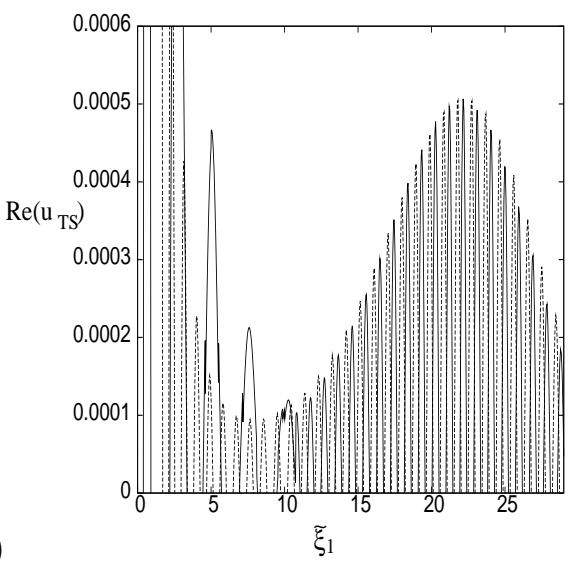

(b)

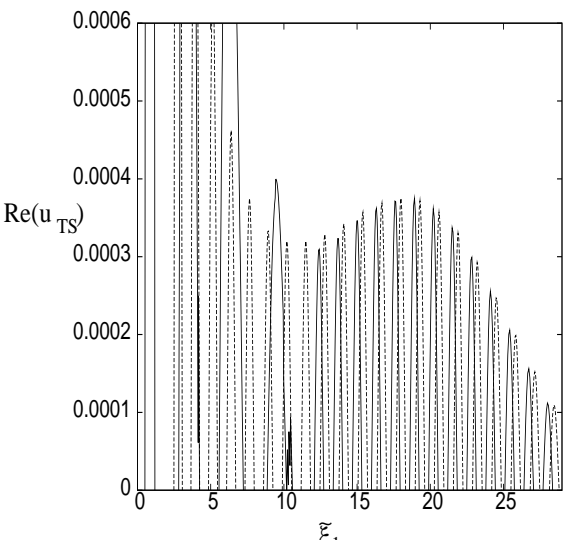

(d)

$\tilde{\xi}_{1}$

Fig. 14 Plot of $K_{\xi}=\left|u_{\mathrm{TS}}\right|$ for (a) $\hat{\sigma}=100$ and (c) $\hat{\sigma}=200$, where the DNS result is given by the solid line and the PSE result by the dashed line. Panels (b) and (d) contain the corresponding plot of $\operatorname{Re}\left(u_{\mathrm{TS}}\right)$. 
For $\hat{\sigma}=100$ and 200 , the function $K_{\xi}=\left|u_{\mathrm{TS}}\right|$, along with $\operatorname{Re}\left(u_{\mathrm{TS}}\right)$, are plotted in figure 14 , for the values of $\tilde{\xi}_{1}^{\mathrm{LE}}$ marked with circles in figure 14 . Here excellent agreement is observed for $K_{\xi}$ around the upper branch point as expected. In the plots of $\operatorname{Re}\left(u_{\mathrm{TS}}\right)$ it is clear to see that the wavelength of the DNS agrees with that of the PSE simulation, in the region where the good agreement for $K_{\xi}$ is achieved. The difference in phase between the two solutions is due to the patching method, but is of little concern here. For smaller values of $\tilde{\xi}_{1}$ the wavelength of the DNS solution is longer than that of the PSE result, but is less than two wavelengths of the $\mathrm{T}-\mathrm{S}$ wave. These figures also show that the minimum value of $K_{\xi}^{\text {DNS }}$ does not correspond to the lower branch point of the PSE method, it actually occurs upstream of this point. The actual minimum value of $K_{\xi}$ for the two methods is very similar, but it is clear that the DNS result is not dominated by the T-S wave until some distance downstream of the lower branch. For the larger value of $\hat{\sigma}$ the distance between the two minimums is smaller, but there are oscillations on $K_{\xi}$ making the determination of a minimum value difficult. By considering the PSE results, the value of $K_{I}(\hat{\sigma})$ for the flat plate can be determined, see figure 15 . This could also be calculated from the DNS using the $e^{N}$ method, see $(\mathbf{7})$. The results give an approximate linear relation for $K_{I}$ where the values are approximately 100 times smaller than those calculated for a Modified Super Ellipse (MSE) by Wanderley \& Corke (37). This clearly shows the importance of considering bodies with non-zero nose curvature, as is done in the next section.

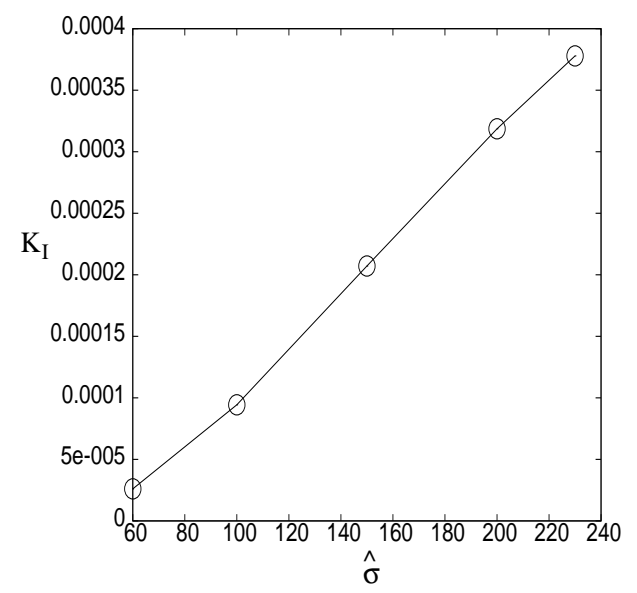

Fig. 15 Plot of $K_{I}$ as a function of $\hat{\sigma}$.

\subsection{A parabolic body}

This section compares results of the PSE and the DNS methods on a parabolic body in order to understand how the introduction of a non-zero nose radius affects the distance at which the non-modal eigenmodes persist in the DNS. In this section the Strouhal number is limited to $S \in[0,0.3]$ as this is a typical range of values found in aircraft. A plot of the 
absolute value of the receptivity coefficient, $C_{1}$ as a function of $S$, can be seen in figure 16 , which is a reproduction of figure $4(\mathrm{a})$ of Hammerton \& Kerschen (16).

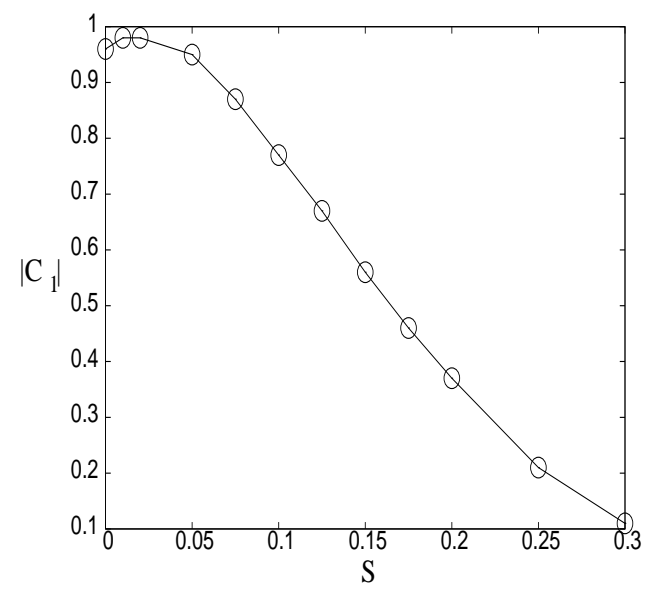

Fig. 16 Plot of $\left|C_{1}\right|$ for a parabolic body as a function of $S$. This is a reproduction of figure 4(a) of Hammerton \& Kerschen (16).
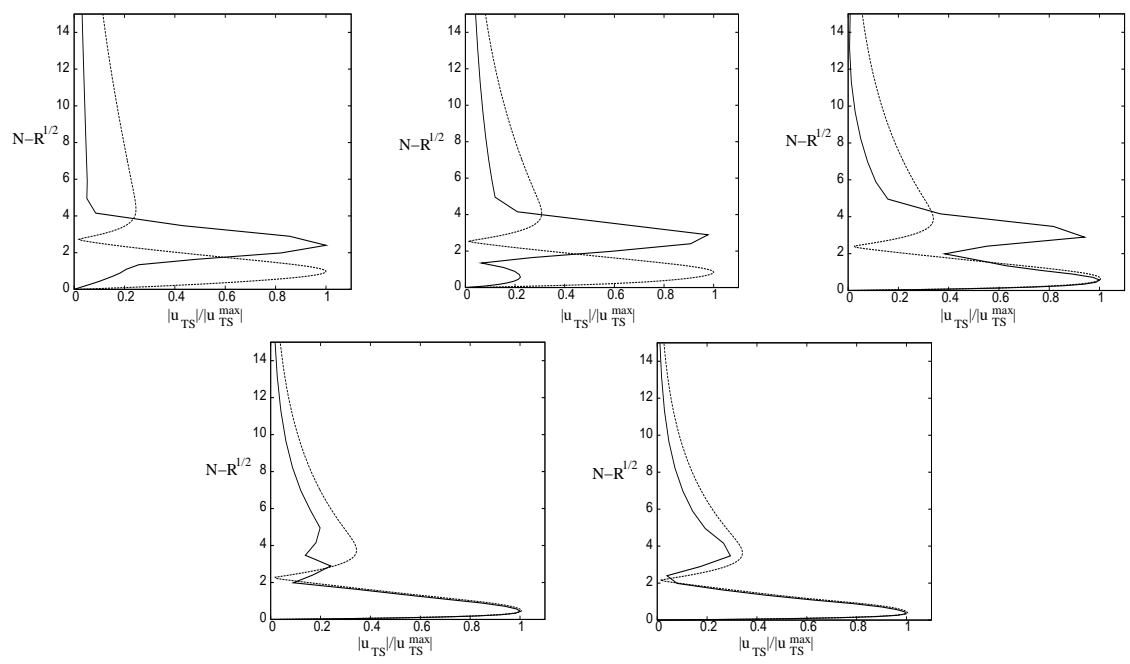

Fig. 17 Plot of the normalized T-S wave profiles $\left|u_{\mathrm{TS}}\right| /\left|u_{\mathrm{TS}}^{\max }\right|$ for $S=0.2$ and $\hat{\sigma}=100$ for $\tilde{\xi}_{1}=5,10,15,20$ and 25 from top left to bottom right. In each panel the solid line gives the DNS result and the dashed line gives the PSE result.

For a parabolic body, there is again a dominant peak in the mode shapes, for small $\tilde{\xi}_{1}$, 
at the edge of the boundary layer, due to either the non-modal eigenmodes or the Stokes layer remnant not being fully removed by the filtering process. This can be seen for $S=0.2$ in figure 17. When these profiles are compared to the $S=0$ result in figure 10, it can be seen in the $\tilde{\xi}_{1}=15$ panel that the non-modal contribution persists further downstream than for the flat plate. Beyond $\tilde{\xi}_{1}=15$ the agreement between the two numerical methods improves, and by $\tilde{\xi}_{1}=25$ the agreement is similar to that of the flat plate case. Here the $\mathrm{T}-\mathrm{S}$ wave now dominates the DNS solution.

(a)

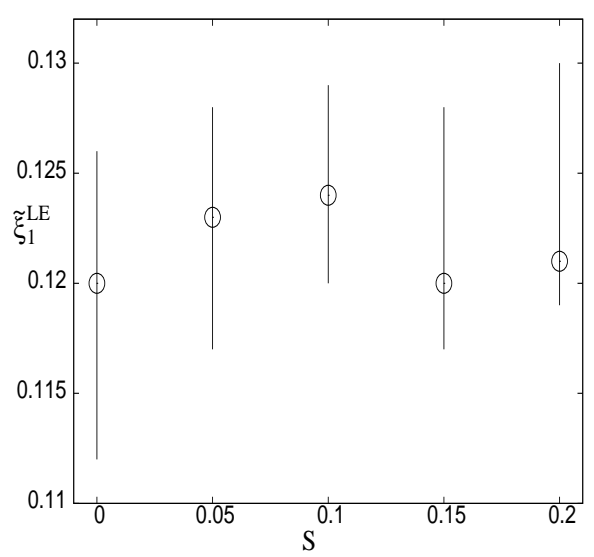

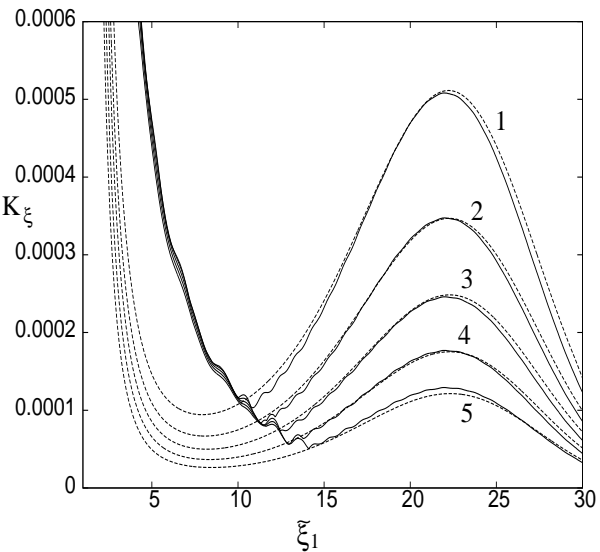

(b)

Fig. 18 Plot of (a) $\tilde{\xi}_{1}^{\mathrm{LE}}(S)$ for $\hat{\sigma}=100$, where the circles indicate the value where $\left|K_{I I}^{\mathrm{PSE}}-K_{I I}^{\mathrm{DNS}}\right|$ is minimised and the solid lines give the $10 \%$ error bars. Panel (b) shows $K_{\xi}$ for $\hat{\sigma}=100$ and $S=0,0.05,0.1,0.15$ and 0.2 numbered $1-5$ respectively. The solid lines are the DNS result and the dashed lines are the PSE result with $\tilde{\xi}_{1}^{\mathrm{LE}}$ given by the circles in panel (a).

Figure 18(a) shows the position of $\tilde{\xi}_{1}^{\mathrm{LE}}$ for the case $\hat{\sigma}=100$. As the Strouhal number varies, the position of $\tilde{\xi}_{1}^{\mathrm{LE}}$ changes only slightly due to the patching procedure. However, if the $10 \%$ error bars are included then it is found that $\tilde{\xi}_{1}^{\mathrm{LE}}$ remains relatively constant as $S$ is increased. If the value of $K_{\xi}$ is now considered in figure 18(b) for the DNS and PSE methods, where $\tilde{\xi}_{1}^{\mathrm{LE}}$ is given by the circles of panel (a), then excellent agreement is found between the two methods for $\tilde{\xi}_{1} \gtrsim 15$. This agrees with the results for the flat plate in figure 14. However, for $\tilde{\xi}_{1}<15$ the non-modal eigenmodes in the DNS dominate the solution, and the lower branch point of the PSE result is upstream of the minimum value of $K_{\xi}$ form the DNS. As the nose radius of the parabola is increased the range over which the non-modal eigenmodes dominate the DNS also increases. Another interesting feature of these results is that, as for the flat plate, the minimum values of $K_{\xi}$ are again approximately 100 times smaller than those for the Modified Super ellipse (MSE) given in Wanderley \& Corke (37). This suggests that the MSE has particular characteristics which give much larger T-S wave amplitudes. The study of these characteristics however, is beyond the scope of this paper. 


\section{Conclusions and discussion}

This paper produced a comparison of the propagation of Tollmien-Schlichting waves on both a semi-infinite flat plate and a parabolic body using the DNS approach of (18) and the PSE method investigated in (19) and (30). It was found that the DNS results contained non-modal eigenmodes or remnants of the Stokes layer, not removed by a filtering process, that dominated the solution a significant distance downstream of the lower branch. The significance of this is that the solution of the PSE method cannot be compared with DNS or experimental results until it is downstream of the lower branch. The exception to this would be if the value of $K_{I}$ were calculated via the DNS using the $e^{N}$ method with a value of $K_{\xi}$ upstream of the lower branch. In this case the PSE approach is likely to give good agreement with this result because it contains only T-S wave amplitude information.

It was also shown that the DNS scheme of (18) does not show the evolution of $\mathrm{T}-\mathrm{S}$ waves upstream of the lower branch neutral stability point as claimed by the authors. The existence of the non-modal eigenmodes of the continuous spectrum meant that the likeness of the mode shapes of the DNS scheme to a T-S wave upstream of the lower branch was a mere coincidence.

Therefore, the PSE scheme here could be used to calculate the growth and propagation of the T-S wave on bodies with modest nose radii. However, if a comparison with DNS or experiments is required, then care would have to be taken that the comparison point is far enough downstream so that the DNS results in the boundary layer were dominated by the $\mathrm{T}-\mathrm{S}$ wave. The results in this paper showed that this point can be a significant distance downstream of the lower branch for free-stream frequencies used in experiments.

Although the PSE method is suitable for calculating T-S wave amplitudes at the lower branch point, care would need to be taken when continuing the calculation to the upper branch point, as nonlinear effects would become significant. In this case the nonlinear version of the PSE (24) should be used.

The author would like to thank Paul Hammerton for his receptivity coefficient data, and for his useful comments and suggestions in the production of this article. This work was carried out while under the support of the EPSRC under grants EP/F069855/1 and EP/G000034/1. Thanks also go to the anonymous referees whose comments and suggestions have lead to an improved manuscript.

\section{References}

1. M. V. Morkovin, Guide to experiments on instability and laminar-turbulent transition in shear layers. Cincinnati, Ohio, 1985.

2. M. E. Goldstein, "The evolution of Tollmien-Schlichting waves near a leading edge," J. Fluid Mech., vol. 127, pp. 59-81, 1983.

3. L.-U. Schrader, L. Brandt, C. Mavriplis, and D. S. Henningson, "Receptivity to freestream vorticity of flow past a flat plate with elliptic leading edge," J. Fluid Mech., vol. 653, pp. 245-271, 2010.

4. M. E. Goldstein, "Scattering of acoustic waves into Tollmien-Schlichting waves by small streamwise variations in surface geometry," J. Fluid Mech., vol. 154, pp. 509-529, 1985.

5. E. J. Kerschen, M. Choudhari, and R. A. Heinrich, "Generation of boundary 
instability waves by acoustic and vortical freestream disturbances," LaminarTurbulent Transition, Vol III. New York: Springer, pp. 477-488, 1990.

6. P. W. Duck, A. I. Ruben, and C. N. Zhikharev, "The generation of Tollmein-Schlichting waves by free-stream turbulence," J. Fluid Mech., vol. 312, pp. 341-371, 1996.

7. X. Wu, "Receptivity of boundary layers with distributed roughness to vortical and acoustic disturbances: a second order asymptotic theory and comparison with experiments," J. Fluid Mech., vol. 431, pp. 91-133, 2001.

8. X. Wu, "On local boyndary-layer receptivity to vortical disturbances in the free stream," J. Fluid Mech., vol. 449, pp. 373-393, 2001.

9. M. E. Goldstein, S. J. Leib, and S. J. Cowley, "Generation of tollmien-schlichting waves on interactive marginally separated flows," J. Fluid Mech., vol. 181, pp. 485-517, 1987.

10. M. E. Goldstein and L. S. Hultgren, "Boundary-layer receptivity to long-wave freestream disturbances," Annu. Rev. Fluid Mech., vol. 21, pp. 137-166, 1989.

11. W. S. Saric, H. L. Reed, and E. J. Kerschen, "Boundary-layer receptivity to freestream disturbances," Annu. Rev. Fluid Mech., vol. 34, pp. 291-319, 2002.

12. S. H. Lam and N. Rott, "Theory of linearized time-dependent boundary layers," Cornell University GSAE Rep. AFOSR, pp. TN-60-1100, 1960.

13. S. N. Brown and K. Stewartson, "On the propagation of disturbances in a laminar boundary layer.," Proc. Camb. phil. Soc, vol. 73, pp. 493-514, 1973.

14. S. H. Lam and N. Rott, "Eigen-functions of linearized unsteady boundary layer equations," Journal of Fluids Engineering, vol. 115, pp. 597-602, 1993.

15. M. E. Goldstein, P. M. Sockol, and J. Sanz, "The evolution of Tollmien-Schlichting waves near a leading edge. Part 2. Numerical determination of amplitudes," J. Fluid Mech., vol. 129, pp. 443-453, 1983.

16. P. W. Hammerton and E. J. Kerschen, "Boundary-layer receptivity for a parabolic leading edge," J. Fluid Mech., vol. 310, pp. 243-267, 1996.

17. D. E. Nichols, "Boundary Layer Receptivity of a Flat Plate with a Rounded Leading Edge," PhD Thesis, University of East Anglia, Norwich, United Kingdom, 2001.

18. O. M. Haddad and T. C. Corke, "Boundary layer receptivity to free-stream sound on parabolic bodies," J. Fluid Mech., vol. 368, pp. 1-26, 1998.

19. M. R. Turner and P. W. Hammerton, "Asymptotic receptivity analysis and the Parabolized Stability Equation : a combined approach to boundary layer transition," J. Fluid Mech., vol. 562, pp. 355-381, 2006.

20. A. Tumin and E. Reshotko, "Spatial theory of optimal disturbances in boundary layers," Phys. Fluids, vol. 13, no. 7, pp. 2097-2104, 2001.

21. R. D. Joslin, C. L. Streett, and C.-L. Chang, "Spatial direct numerical simulation of boundary-layer transition mechanisms: Validation of PSE theory," Theoret. Comput. Fluid Dynamics, vol. 4, pp. 271-288, 1993.

22. M. Gaster, "On the effects of boundary-layer growth on flow stability," J. Fluid Mech., vol. 66, pp. 465-480, 1974.

23. W. Saric and A. Nayfeh, "Nonparallel stability of boundary-layer flows," Phys. Fluids, vol. 18, pp. 945-950, 1975.

24. F. P. Bertolotti, T. Herbert, and P. R. Spalart, "Linear and nonlinear stability of the Blasius boundary layer," J. Fluid Mech, vol. 242, pp. 441-474, 1992.

25. J. L. Van Ingen, "The $e^{N}$ method for transition prediction. Historical review of work at 
TU Delft," AIAA Paper 2008-3830, 2008.

26. M. Langlois, G. Casalis, and D. Arnal, "On the Practical Application of the PSE Approach to Linear Stability Analysis," Aerospace Science and Technology, vol. 2, no. 3, pp. 167-176, 1998 .

27. C. Chang, "The Langley Stability and Transition Analysis Code (LASTRAC): LST, Linear \& nonlinear PSE for 2-D, axisymmetric, and infinite swept wing boundary layers," AIAA Paper, vol. 974, p. 2003, 2003.

28. P. Andersson, D. S. Henningson, and A. Hanifi, "On a stabilization procedure for the parabolic stability equations," J. Engrg. Math., vol. 33, no. 3, pp. 311-332, 1998.

29. M. R. Turner, "Numerical and Asymptotic Approaches to Boundary-Layer Receptivity and Transition," PhD Thesis, University of East Anglia, Norwich, United Kingdom, 2005.

30. M. R. Turner and P. W. Hammerton, "Analysis of the unstable Tollmien-Schlichting on bodies with a rounded leading edge using the parabolized stability equation," $J$. Fluid Mech., vol. 623, pp. 167-185, 2009.

31. H. L. Reed, N. Lin, and W. S. Saric, "Boundary layer receptivity to sound: Navier-Stokes computations," Appl. Mech. Rev., vol. 43, no. 5, pp. S175-S180, 1990.

32. W. S. Saric and B. K. Rasmussen, "Boundary layer receptivity: Freestream sound on an elliptic leading edge," Bull. Am. Phys. Soc., vol. 37, p. 1720, 1992.

33. W. S. Saric, W. Wei, and B. K. Rasmussen, "Effect of leading-edge on sound receptivity," Laminar-Turbulent Transition, (IUTAM Sendai, 1994, ed. R. Kobayashi), Springer, 413-420, 1995.

34. N. Lin, H. L. Read, and W. S. Saric, Effect of leading-edge geometry on boundarylayer receptivity to free-stream sound, Instability, Transition and Turbulence. Berlin: Springer, 421-440, 1992.

35. E. Erturk and T. C. Corke, "Boundary layer receptivity to sound at incident angles," J. Fluid Mech., vol. 444, pp. 383-407, 2001.

36. O. M. Haddad, E. Erturk, and T. C. Corke, "Acoustic receptivity of the boundary layer over parabolic bodies at angles of attack," J. Fluid Mech., vol. 536, pp. 377-400, 2005.

37. J. B. V. Wanderley and T. C. Corke, "Boundary layer receptivity to free-stream sound on elliptic edges of flat plates," J. Fluid Mech., vol. 429, pp. 1-21, 2001.

38. D. A. Fuciarelli, H. L. Reed, and I. Lyttle, "DNS of leading-edge receptivity to sound," AIAA Paper 98-2644, 1998.

39. W. S. Saric and E. B. White, "Influence of high-amplitude noise on boundary-layer transition to turbulence," AIAA Paper 98-2645, 1998.

40. Rosenhead, Laminar Boundary Layers. An account of the development, structure and stability of laminar boundary layers in incompressible fluids, together with a description of the associated experimental techniques. Clarendon Press, Oxford, 1963.

41. R. T. Davis, "Numerical solution of the navier-stokes equations for symmetric laminar incompressible flow past a parabola," J. Fluid Mech., vol. 51, pp. 417-433, 1972.

42. O. M. Haddad, "Numerical study of leading edge receptivity over parabolic bodies," PhD Thesis, Illinois Institute of Technology, Illinois, USA, 1995.

43. D. A. Anderson, J. C. Tannehill, and R. H. Pletcher, "Computational Fluid Mechanics and Heat Transfer. (Chapter 5)," McGraw-Hill (New York), 1984.

44. B. G. B. Klingmann, A. V. Boiko, K. J. A. Westin, V. V. Kozlov, and P. H. Alfredsson, "Experiments on the stability of tollmien-schlichting waves," Eur. J. Mech. B, vol. 12, 
pp. 493-514, 1993.

45. P. W. Hammerton, "Comparison of Lam-Rott and Brown-Stewartson eigensolutions of the boundary-layer equations," Quart. J. Mech. Appl. Math., vol. 52, no. 3, pp. 373385, 1999.

46. R. L. Panton, Incompressible flows. John Wiley (New York), 1984. 